\title{
AN IRAS-BASED SEARCH FOR NEW DUSTY LATE-TYPE WC WOLF-RAYET STARS
}

\author{
MARTIN COHEN \\ Radio Astronomy Laboratory, University of California, Berkeley \\ Received 1994 November 9; accepted 1995 March 23
}

\begin{abstract}
I have examined all Infrared Astronomical Satellite (IRAS) data relevant to the 173 Galactic Wolf-Rayet (W-R) stars in an updated catalog, including the 13 stars newly discovered by Shara and coworkers. Using the W-R coordinates in these lists, I have examined the IRAS Point Source Catalog ( PSC), the Faint Source Catalog, and the Faint Source Reject Catalog, and have generated one-dimensional spatial profiles ("ADDSCANs") and two-dimensional full-resolution images ("FRESCOs"). The goal was to assemble the best set of observed IRAS color indices for different W-R types, in particular for known dusty late-type WC Wolf-Rayet (WCL) objects. I have also unsuccessfully sought differences in $I R A S$ colors and absolute magnitudes between single and binary W-R stars. The color indices for the entire ensemble of W-R stars define zones in the IRAS color-color ([12] [25], [25] - [60])-plane. By searching the PSC for otherwise unassociated sources that satisfy these colors, I have identified potential new $\mathrm{W}-\mathrm{R}$ candidates, perhaps too faint to have been recognized in previous optical searches. I have extracted these candidates' IRAS low-resolution spectrometer (LRS) data and compared the spectra with the highly characteristic LRS shape for known dusty WCL stars. The 13 surviving candidates must now be examined by optical spectroscopy. This work represents a much more rigorous and exhaustive version of the LRS study that identified IRAS $17380-3031$ (WR98a) as the first new W-R (WC9) star discovered by IRAS (Cohen and coworkers). This search should have detected dusty WCL stars to a distance of $7.0 \mathrm{kpc}$ from the Sun, for $|l|>30^{\circ}$, and to $2.9 \mathrm{kpc}$ even in the innermost Galaxy. For free-free-dominated W-R stars the corresponding distances are 2.5 and $1.0 \mathrm{kpc}$, respectively.
\end{abstract}

Subject headings: circumstellar matter - infrared: stars — stars: Wolf-Rayet — surveys

\section{INTRODUCTION}

Fewer than 200 Galactic Wolf-Rayet (W-R) stars are currently known (cf. van der Hucht et al. 1988, hereafter HHASD), all but four discovered by optical techniques. Shara et al. (1991, hereafter SPMS) have demonstrated significant incompleteness of the sample of known W-R stars even at distances as close as $2 \mathrm{kpc}$ from the Sun. They estimate that this incompleteness attains $25 \%$ for the $2-3 \mathrm{kpc}$ range. Traditional photographic techniques for seeking W-R stars have been superseded by more efficient methods using CCD detectors and narrow passbands designed to isolate specific emission lines or line complexes (e.g., SPMS). Nevertheless, all these techniques are still optically based. While they offer the advantage of reduced confusion in regions of high star density, they are still subject to the vagaries of interstellar extinction. It is, therefore, worthwhile to consider whether the infrared offers a viable alternative in the quest for new $W-R$ stars. While infrared (longward of $2 \mu \mathrm{m}$ ) spectroscopy of W-R stars has been pursued (e.g., Eenens, Williams, \& Wade 1991), it has not yet developed into a survey tool to seek new W-R stars. However, the IRAS survey affords an extinction of only about $4.5 \%$ of $A_{V}$ at its shortest wavelength $(12 \mu \mathrm{m})$, and essentially negligible extinction at 25,60 , and $100 \mu \mathrm{m}$.

Late-type WC W-R stars (WCLs) frequently have circumstellar dust shells (e.g., Cohen, Barlow, \& Kuhi 1975; Gehrz \& Hackwell 1974; Williams, van der Hucht, \& Thé 1987, hereafter WHT), whose thermal emission dominates both stellar photosphere and free-free emission from stellar winds. This phenomenon becomes more common with lateness of WC subtype. Dust shells can appear as early as type WC4, but only episodically, whereas dust shells are persistent phenomena in the large majority of WC9s studied (WHT). One might well wonder whether dusty WCLs (DWCLs) have been preferentially overlooked or are even invisible in previous optical searches for W-R stars owing to their often substantial circumstellar extinctions (e.g., Cohen \& Kuhi (1977a) for Ve 2-45 and GL 2104). Indeed, the most recently discovered WC8WC10 stars were all recognized in infrared surveys, namely, GL 2104 and GL 2179 (Cohen \& Kuhi 1976, 1977b; Allen et al. 1977) from the AFGL Rocket Sky Survey (Price \& Walker 1976); WR 48a by Danks et al. (1983); and IRAS 17380-3031 (Cohen et al. 1991). Clearly, a systematic infrared search would provide a valuable adjunct to conventional W-R search techniques.

WCLs (particularly WC9s) show a curious concentration toward the Galactic center (Smith 1968). Newer catalogs of W-R stars (HHASD; Conti \& Vacca 1990) have reinforced this early discovery: there is an essentially monotonic trend toward higher Galactocentric radius for the outer edge of the zones containing earlier WC subtypes. Smith \& Maeder (1991) explain this confinement of the WCL subtypes to the inner Galaxy in terms of the influence of local initial metallicity $(Z)$ on high-mass stellar evolution: the lower $Z$, the higher the surface composition ratio of $(\mathrm{O}+\mathrm{C}) / \mathrm{He}$ when the star becomes a $W C$, hence the earlier the type (at low $Z$, radiatively driven mass loss is reduced [Abbott 1982; Kudritzki, Pauldrach. \& Puls 1987], and newly made $C$ and $O$ are revealed at the surface later in the evolution). Smith \& Maeder argue that only in 

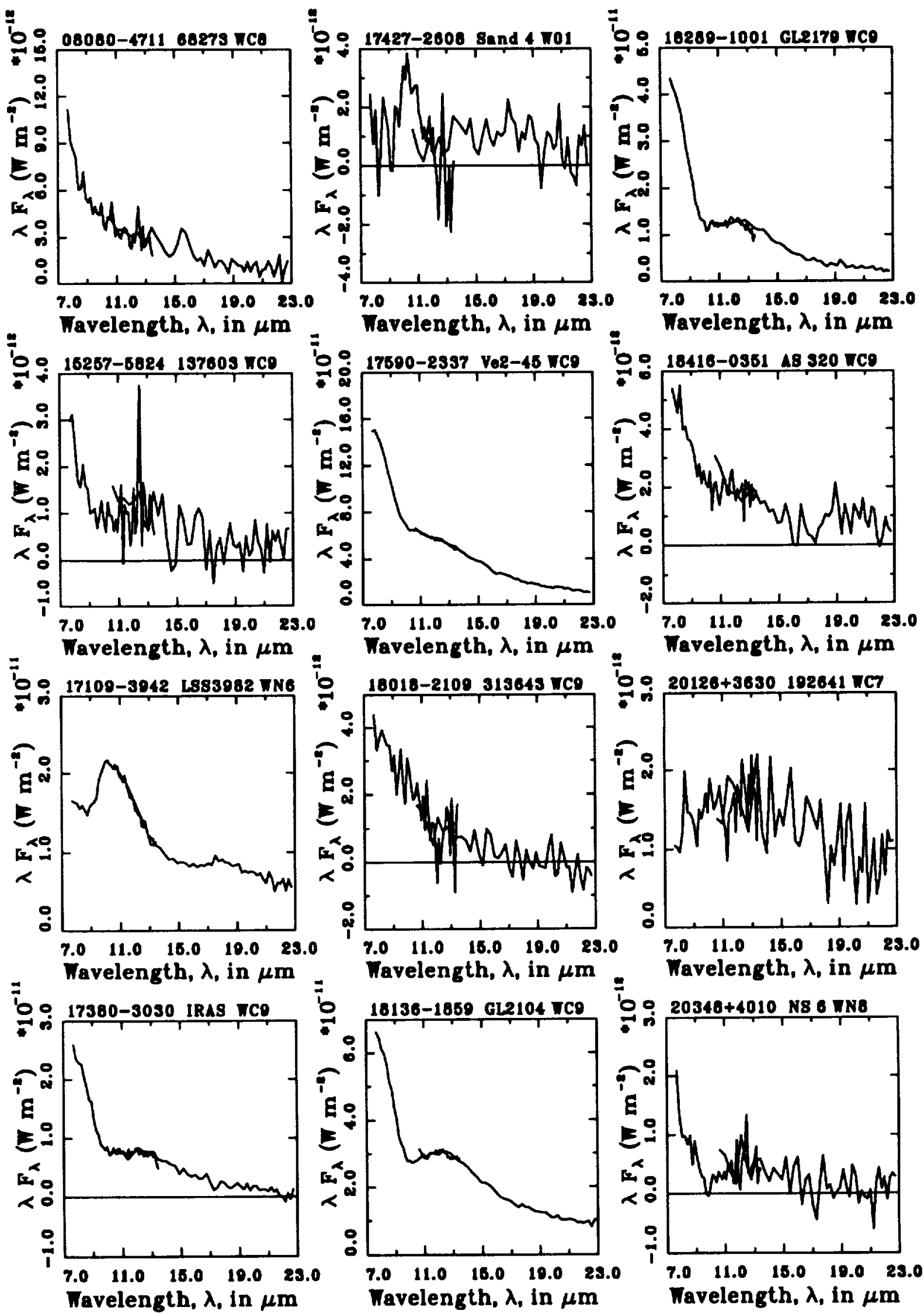

FIG. 1.-Montage of useful LRS spectra for known W-R stars in the form of $\lambda F_{\lambda}$ plots 
regions of the highest metallicity is it possible to create WCL stars. The combination of new surface abundances with the latest stellar models yields such an elegant explanation for this odd distribution (even given one counterexample) that it merits further study. WC9s, in particular, represent the lowest mass and coolest known W-R stars. It is precisely toward the Galactic center that conventional optical searches for W-R stars would be expected to fail. By contrast, $I R A S$ provides an ideal database in which to hunt for W-R stars because of $(1)$ its reduced sensitivity to interstellar extinction close to the Galactic plane, where W-R stars are located; (2) its sensitivity to DWCLs with their infrared-bright dust shells; and (3) its sky coverage ( $96 \%$ complete over the entire sky).

Early photometry of W-R stars from IRAS was reported by van der Hucht et al. (1985a), along with spectra from the Dutch low-resolution spectrometer on IRAS (Atlas of Low Resolution IRAS Spectra 1986, hereafter LRS). The LRS can play a potentially important role in a search for new DWCLs, as discussed below. Indeed, the LRS spectrum of IRAS 17380-3031 was the sole criterion for suggesting that this bright $I R A S$ source $\left(S_{12} \sim 50 \mathrm{Jy}\right.$ ) might be a DWCL (Volk \& Cohen 1989), a suggestion only much later vindicated by optical spectroscopy (Cohen et al. 1991).

In the present paper I describe an attempt to find new W-R stars of all types, using an approach based entirely on IRAS data but emphasizing DWCLs. Thirteen new DWCL candidates are proposed but must await either optical or near-infrared spectroscopy to test the proposal. Section 2 outlines the role of the LRS; $\S 3$ details the IRAS data for W-R stars that were analyzed; $\S 4$ summarizes the $I R A S$ color-color analyses of W-R stars; $\S 5$ describes the actual search criteria used to isolate new W-R stars and the candidates identified for future study; $\S 6$ examines the average $I R A S$ energy distributions of W-R stars in terms of the emission mechanisms; and $\S 7$ treats the likely completeness of these searches on the basis of the absolute magnitudes for W-R stars dominated by either of the two basic emission processes (dust and free-free).

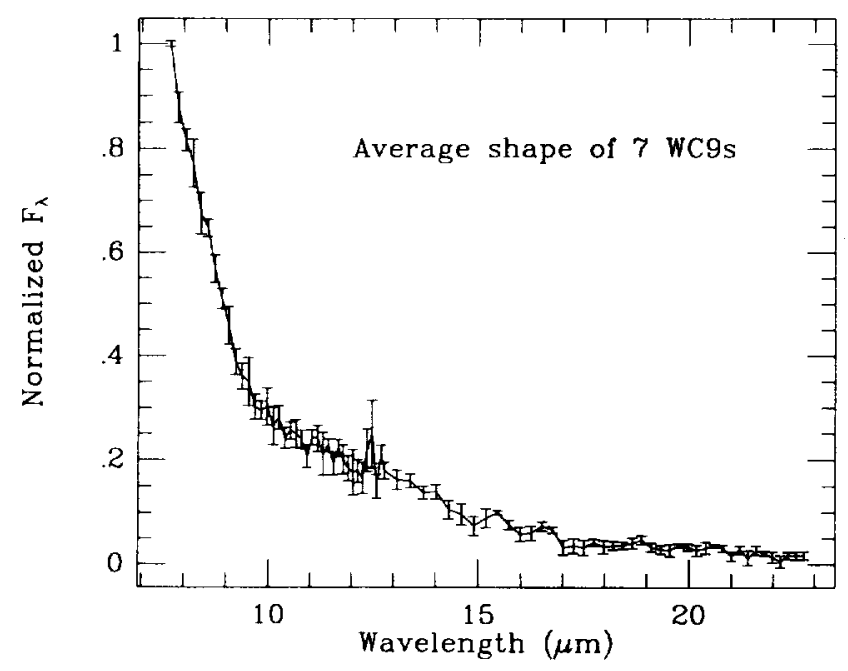

FIG. $2 a$

\section{THE ROLE OF THE LRS}

Working from coordinates for the established Galactic W-R stars given by HHASD, including the 13 stars found by SPSM and IRAS $17380-3031$, I searched the LRS database for positional matches. This LRS survey of all known W-R stars reveals few useful spectra, essentially because W-R stars inhabit the Galactic plane, where IRAS suffers the greatest confusion by high source density. The LRS, incidentally, provides a powerful means of rejecting spurious matches, namely, a bright $M$ giant lying within 1' of the position of WR $85=$ LSS 3982 , because LRS spectral shapes of different categories of star are already quite well known (cf. Volk \& Cohen 1989). The census of 11 relevant and meaningful LRS spectra for known W-R stars is intriguing: one WO1, one WN8, one WC7, one WC8. and seven WC9s. Figure 1 presents these LRS spectra. Even a cursory examination of the DWCL spectra (for AS 320, HDE 313643, GL 2104, IRAS 17380-3031, GL 2179, Ve 2-45, and HD 137603) suggests that these seven WC9s exhibit startlingly similar spectral shapes. This impression is borne out quantitatively by Figure $2 a$, displaying the average of the seven individual LRS spectra (normalized to unity before averaging). The dispersion in shape is quite small and can be enhanced by combining only those spectra with the highest signal-to-noise ratios, namely, those for GL 2104, IRAS 17380-3031, GL 2179 , and Ve $2-45$ (Fig. $2 b$ ). The dispersion in shape now has an average of only $3.0 \%$. Using a qualitative version of this technique, Volk \& Cohen (1989) argued that IRAS 17380-3031 was a DWCL. Figure 1 shows just how very much like the DWCL IRS spectra known at that time is the LRS spectrum of IRAS 17380-3031. Furthermore, this shape does not typify any other kind of astronomical object, indicating that it might provide a unique criterion for recognizing DWCLs. The shape has been explained by Cohen, Tielens, \& Bregman (1989) as a combination of broad 7-9 $\mu \mathrm{m}$ emission by polyaromatic carbon clusters and the effects of interstellar extinction near $10 \mu \mathrm{m}$.

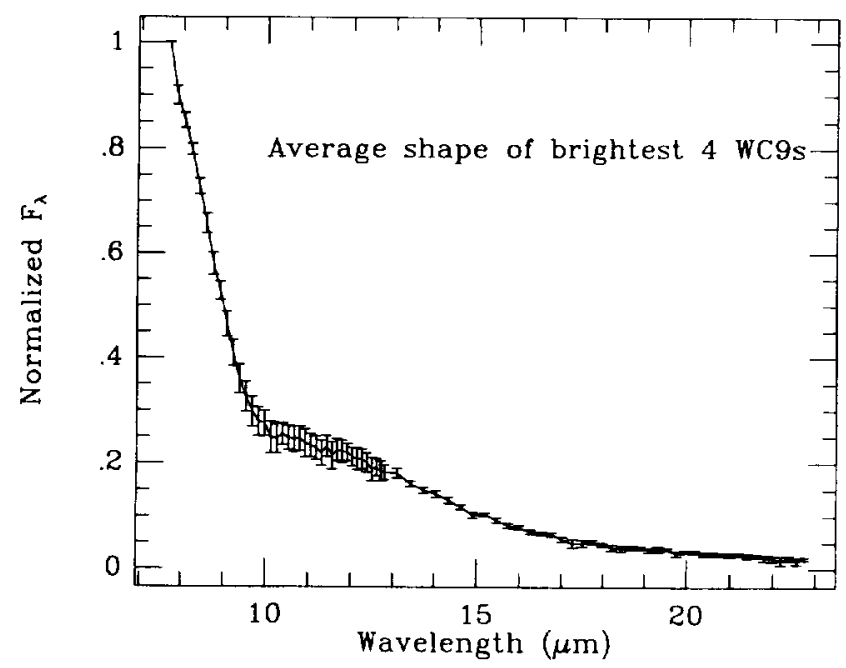

FIG. $2 b$

FIG. 2.-Average of normalized LRS spectra with error bars representing standard errors of the mean. $(a)$ For all seven DWCLs. $(b)$ For the four DWCLs with highest signal-to-noise ratios in their LRS spectra. 
The character of the LRS spectra for the non-WC9 stars shows the following: for HD $68273\left(\gamma^{2} \mathrm{Ve}\right.$ [WC8]) a spectrum dominated by free-free emission with a clearly detected [Ne III] emission line (near $15.6 \mu \mathrm{m}$ : see also Barlow, Roche, \& Aitken 1988; van der Hucht \& Olnon 1985); for Sanduleak 4 (WOI) the spectrum is equivocal but may also suggest freefree emission from the powerful stellar wind; for HD 192641 (WC7) the spectrum is evidently dominated by thermal emission from circumstellar dust grains; and for NS $6=$ AS 431 (WN8) the spectrum appears to show free-free radiation from a stellar wind, with an overlying silicate absorption feature, essentially in accord with the interpretation of the radio and optical spectra (Caillault et al. 1985). Volk et al. (1991, 1992) have extended Volk \& Cohen's (1989) flux-limited $\left(S_{12} \geq 40\right.$ Jy) sample of LRS spectra to IRAS thresholds of 20 and 10 Jy. Browsing through the resulting catalogs of spectra for the additional 2652 sources did suggest some possible DWCL-like shapes, but none so convincing in character as that of IRAS 17380-3031. Efforts to enlarge the sample of DWCLs by isolating more IRAS sources ostensibly with the "same" characteristic LRS shape have been entirely unsuccessful (M. Cohen 1992, unpublished Anglo-Australian Telescope [AAT] spectroscopy). The likeliest candidate, akin to IRAS 17380-3031, was IRAS 18405-0448, also suggested by Volk \& Cohen (1989). But its optical and near-infrared spectra (Williams et al. 1995) indicate an extreme emission-line star, definitely not a WCL star. It seemed obvious that a method for sharpening the IRAS source selection criteria was needed, rather than systematic browsing of thousands of LRS spectra to ever-decreasing flux density levels (which alternative, of course, is still being pursued). The LRS could play a valuable role in recognizing new DWCLs if one could sharply restrict the number of IRAS sources whose spectra one must examine.

\section{THE $I R A S$-BASED STRATEGY AND DATA ANALYZED}

Walker et al. (1989) have described a practical color-color scheme for separating different categories of astronomical object solely by their $I R A S$ attributes. Their "occupation zones" are illustrated by the unhatched boxes in Figure 3 for the IRAS $[12]-[25]-[60]$ plane. Each such zone includes $70 \%$ of the relevant population of known astronomical sources assembled to define the population's IRAS characteristics, so that the zones do overlap, somewhat, outside the box boundaries. However, the hope was that, if one could define a suitably restricted zone corresponding to W-R stars, one might use the IRAS broadband colors alone to isolate a sample of sources for subsequent LRS analysis.

The logical route was to assemble every piece of $I R A S$ data on all known W-R stars and extract the quintessential attributes for the class. The easiest searches were those through the IRAS Point Source Catalog Version 2 (1988, hereafter PSC) and Faint Source Survey databases because relational queries could be run. The PSC and LRS (1986) have existed for many years as relational databases, accessed by INGRES (PSC) or software from SRON, Groningen (LRS), and maintained at the NASA-Ames Research Center. I queried the PSC for all sources within 2' of the exact coordinates of all the known $\mathrm{Ga}$ lactic W-R stars in an updated catalog provided by van der Hucht (1991). The Faint Source Survey represents the most

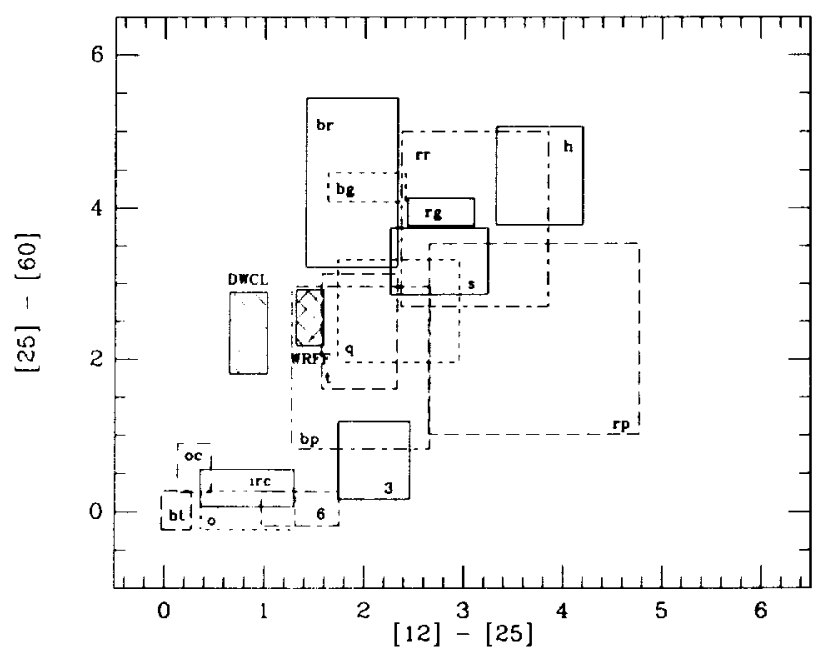

FIG. 3.- "Occupation zones" characterizing different types of source in the $I R A S[12]-[25]-[60] \mu \mathrm{m}$ color-color plane. The letter key to different source categories is a more transparent one than that by Walker et al. (1989), but the same zones are represented. Key: "bt" = bright normal stars; "o" = O-rich giants; " $\alpha$ " = optically visible $\mathrm{C}$ giants; "irc" = infrared-detectable C giants; " 3 " and " 6 " refer to IRAS sources with "LRSCHAR" = 3 or 6 ; "bp" and "rp" = blue and red planetaries; " $t$ " = T Tauri stars; "q" = quasars; " $s$ " = Seyferts; "bg" and "rg" = blue and red galaxies; "br" and "rr" = blue and red reflection nebulae; " $h$ " = H-II regions. The occupation zone corresponding to DWCLs is indicated by the hatched box to the left of the plane; the zone corresponding to all other WRs is the cross-hatched box labeled "WRFF."

reliable sources from the entire Faint Source data set (which results from co-adding $I R A S$ data from all the separate coverages of the sky), namely, those at preferentially higher Galactic latitudes where confusion is not an issue. At lower latitudes, extracted point sources are passed to the Faint Source Reject Catalog. Both the Faint Source Catalog (FSC) and the Faint Source Reject File (FSRF) (Moshir et al. 1989) exist as relational databases at IPAC, accessed by SYBASE. These were also searched using the same radius as for the PSC. Useful data were found on 64,1 , and $61 \mathrm{~W}$-R stars in the PSC, FSC, and FSRF, respectively.

Because relational searches necessarily involve some limiting radius, there is always the question of whether a "matched" source is truly the W-R star. Therefore, I also examined twodimensional images that preserved the full spatial resolution of each IRAS band, for all W-R stars, at all four wavelengths ("FRESCOs"). Given accurate coordinates, the most reliable way to detect the faintest matches to W-R stars comes from coadding all the one-dimensional IRAS crossings through the W-R star's position ("ADDSCANs"), using two-dimensional images to help disentangle neighboring sources. The several resulting data sets were combined into a unified $I R A S$ database for known W-R stars from which IRAS attributes could be defined for each of the WN and WC subtypes as well as for the entire class of W-R stars.

Table 1 presents all the data acquired from the IRAS catalogs, and indicates the source of each datum ("P" = PSC; "F" = Faint Source Catalog or Reject File; "A" = ADDSCAN; "FR" = FRESCO). In the absence of detections, $3 \sigma$ upper limits to flux densities are given. In this context, FRESCO in- 
TABLE 1

IRAS Photometry for Galactic Wolf-Rayet Stars (arranged by HHASD number)

\begin{tabular}{|c|c|c|c|c|c|c|c|}
\hline$\overline{\mathrm{WR}}$ & Name(HD) & Spectrum & $\begin{array}{l}\text { Data } \\
\text { Type }\end{array}$ & $\begin{array}{l}\text { S12 } \\
\text { [Jy] }\end{array}$ & $\begin{array}{l}525 \\
\text { [Jy] }\end{array}$ & $\begin{array}{l}560 \\
\text { [Jy] }\end{array}$ & $\begin{array}{l}\mathrm{S100} \\
{[\mathrm{Jy}]}\end{array}$ \\
\hline \multirow[t]{3}{*}{1} & 4004 & WN5 & $\mathrm{A}$ & 0.255 & 0.12 & $<0.51$ & $<3.0$ \\
\hline & & & $\mathbf{F}$ & 0.252 & 0.184 & $<0.80$ & $<9.6$ \\
\hline & & & FR & 0.217 & 0.076 & $<0.62$ & $<3.3$ \\
\hline \multirow[t]{2}{*}{2} & 6327 & WN2 & $\mathbf{F R}$ & 0.069 & 0.083 & $<2.4$ & $<8.3$ \\
\hline & & & $\mathbf{A}$ & 0.033 & 0.075 & 0.17 & \\
\hline \multirow[t]{2}{*}{3} & 9974 & WN3+a(SB1) & $\mathbf{A}$ & $<0.04$ & 0.038 & 0.080 & $<0.29$ \\
\hline & & & FR & 0.027 & 0.032 & 0.038 & $<1.4$ \\
\hline \multirow[t]{2}{*}{4} & 16523 & WC5(SB1) & $\mathbf{A}$ & 0.10 & 0.065 & 0.04 & $<2.4$ \\
\hline & & & FR & 0.110 & 0.059 & 0.034 & $<1.2$ \\
\hline \multirow[t]{2}{*}{5} & 17638 & WC6 & A & 0.16 & 0.07 & $<0.40$ & $<5.0$ \\
\hline & & & FR & 0.261 & 0.038 & $<0.35$ & $<1.5$ \\
\hline \multirow[t]{4}{*}{6} & 50896 & WN5(SB1) & $\mathbf{A}$ & 1.06 & 0.58 & 0.82 & $<2.7$ \\
\hline & & & $\mathbf{P}$ & 1.07 & 0.62 & 0.81 & $<4.8$ \\
\hline & & & $F$ & 1.00 & 0.615 & $0.66:$ & $<1.8$ \\
\hline & & & FR & 0.79 & 0.50 & 0.62 & $<4.5$ \\
\hline \multirow[t]{4}{*}{7} & 56925 & WN4 & $\mathbf{P}$ & $<0.29$ & 0.49 & 9.45 & 16.9: \\
\hline & & & $F$ & $<0.14$ & 0.52 & $<10$. & $<160$ \\
\hline & & & A & 0.11 & 0.35 & & \\
\hline & & & FR & $<3.3$ & 4.33 & cnfsd. & cnfsd. \\
\hline \multirow[t]{3}{*}{8} & 62910 & WC4/WN6 & $\mathbf{A}$ & 0.12 & 0.16 & $<1.3$ & $<2.5$ \\
\hline & & & $\mathbf{F}$ & $<0.08$ & $<0.13$ & 0.99 & $<6.8$ \\
\hline & & & FR & 0.126 & 0.305 & cnfsd. & cnfsd. \\
\hline \multirow[t]{3}{*}{9} & 63099 & WC5+O7 & $\mathbf{A}$ & 0.155 & 0.071 & 0.08 & $<0.9$ \\
\hline & & & $\mathbf{F}$ & 0.126 & $<0.14$ & $<0.7$ & $<6.3$ \\
\hline & & & FR & 0.141 & 0.063 & 0.34 & $<4.4$ \\
\hline \multirow[t]{2}{*}{10} & 65865 & WN4.5 & FR & 0.108 & 0.064 & cnfsd. & cnfsd. \\
\hline & & & $A$ & 0.110 & 0.150 & 0.122 & \\
\hline \multirow[t]{4}{*}{11} & 68273 & WC8+O9I & A & 19.20 & 8.14 & 4.36 & 3.07 \\
\hline & & & $\mathbf{F}$ & 20.16 & 8.71 & 4.26 & $<7.5$ \\
\hline & & & $\mathrm{P}$ & 19.37 & 8.67 & 4.26 & $<13$ \\
\hline & & & FR & 15.53 & 7.95 & 3.49 & $<7.5$ \\
\hline \multirow[t]{2}{*}{12} & CD-45 4482 & WN7(SB1) & FR & 0.085 & 0.048 & cnfsd. & cnfsd. \\
\hline & & & $\mathbf{A}$ & 0.155 & 0.118 & & \\
\hline 13 & Ve $6-15$ & WC6 & $\begin{array}{l}\text { FR } \\
\text { A }\end{array}$ & $\begin{array}{c}\text { cnfsd. } \\
0.072\end{array}$ & $\begin{array}{c}\text { cnfsd. } \\
0.059\end{array}$ & cnfsd. & cnfid. \\
\hline 14 & 76536 & WC6 & A & $<5.2$ & $<8.7$ & $<2.9$ & $<21$ \\
\hline 15 & & & $\begin{array}{l}\text { FR } \\
\text { A }\end{array}$ & $\begin{array}{c}\text { cnfsd. } \\
0.64\end{array}$ & $\begin{array}{c}\text { cnfsd. } \\
0.31\end{array}$ & $\begin{array}{c}\text { cnfsd. } \\
1.50\end{array}$ & cnfsd. \\
\hline 15 & 19013 & weo & $\mathbf{P}$ & 0.56 & $0.23:$ & $1.76:$ & $<21$. \\
\hline & & & $\mathbf{F}$ & 0.64 & $<0.5$ & $<6.3$ & $<66$ \\
\hline & & & FR & 0.69 & 0.65 & 1.68 & $<42$ \\
\hline 16 & 86161 & WN8 & $\mathbf{P}$ & 0.52 & 0.30 & $<1.3$ & $<26$ \\
\hline & & & $\mathbf{A}$ & 0.48 & 0.24 & & \\
\hline & & & $\mathbf{F}$ & 0.45 & $<0.6$ & $<9.7$ & $<27$ \\
\hline & & & FR & 0.45 & 0.030 & $<13$ & cnfsd. \\
\hline 17 & 88500 & WC5 & FR & 0.109 & 0.027 & $<0.6$ & $<1.2$ \\
\hline & & & A & 0.031 & 0.060 & & \\
\hline 18 & 89358 & WN5 & FR & $<3.2$ & $<7.7$ & $\ldots \ldots$ & $<130$ \\
\hline & & & A & 0.43 & 0.27 & & \\
\hline 19 & LS 3 & WC4 & $\begin{array}{l}\text { FR } \\
\text { A }\end{array}$ & $\begin{array}{l}<4.2 \\
0.27\end{array}$ & $\begin{array}{l}<11 . \\
0.34\end{array}$ & $<140$ & $<280$ \\
\hline $19 a$ & SMSP 1 & WN7 & $\mathbf{F}$ & $<9.8$ & $<9.3$ & $<160$ & $<300$ \\
\hline & & & FR & $<40$ & $<110$ & $<1200$. & $<1400$ \\
\hline 20 & BS 1 & WN4.5 & FR & $<3.1$ & $<3.3$ & $<35$ & $<78$ \\
\hline & & & A & 0.076 & 0.079 & 0.42 & 1.00 \\
\hline $20 a$ & SMSP 2 & WN7 & FR & cnfsd. & cnfsd. & cnfsd. & cnfsd. \\
\hline $20 \mathrm{~b}$ & SMSP 3 & WN7 & FR & cnfsd. & cnfsd. & cnfsd. & cnfsd. \\
\hline 21 & 90657 & WN4+04-6 & $\mathbf{P}$ & $<0.71$ & $<2.2$ & $<3.4$ & $<26$ \\
\hline & & & A & 0.27 & & & \\
\hline & & & FR & $<3.8$ & $<2.5$ & $<42$. & $<66$. \\
\hline 22 & 92740 & WN7+a(SB1) & $\mathbf{A}$ & 1.80 & $<8.7$ & cnfsd. & cnfsd. \\
\hline & & & $\mathbf{F}$ & $<22$. & $<21$ & $<103$ & $<770$ \\
\hline & & & FR & $<41$. & $<162$ & cnfsd. & enfsd. \\
\hline 23 & 92809 & WC6 & FR & $<28$ & $<36$ & $<400$ & $<1300$ \\
\hline 24 & 93131 & WN7+a & FR & $<210$ & $<1800$ & $<5900$. & $<4000$ \\
\hline 25 & 93162 & $\mathrm{WN} 7+04 \mathrm{f}$ & $F$ & $<49$ & $<360$ & $<280$ & $<10500$. \\
\hline
\end{tabular}


TABLE I-Continted

\begin{tabular}{|c|c|c|c|c|c|c|c|}
\hline WR & Name(HD) & Spectrum & $\begin{array}{l}\text { Data } \\
\text { Type }\end{array}$ & $\begin{array}{l}\text { S12 } \\
{[\mathrm{Jy}]}\end{array}$ & $\begin{array}{l}\text { S25 } \\
{[\mathrm{Jy}]}\end{array}$ & $\begin{array}{l}\text { S60 } \\
{[\mathrm{Jy}]}\end{array}$ & $\begin{array}{c}\mathbf{S 1 0 0} \\
{[\mathrm{Jy}]}\end{array}$ \\
\hline & & & FR & cnfsd. & cnfsd. & cnfsd. & cnfsd. \\
\hline 26 & MS 1 & WCE/WN5 & FR & $<8.3$ & $<4.3$ & $<28$ & $<88$ \\
\hline \multirow[t]{2}{*}{27} & $\operatorname{LS} 4$ & WC6+a & $\mathbf{F}$ & $<2.9$ & $<3.9$ & $<560$ & $<5200$ \\
\hline & & & FR & $<31$. & $<74$ & $<730$ & $<840$ \\
\hline \multirow[t]{2}{*}{28} & MS 2 & WN7 & $\mathbf{A}$ & 0.60 & 1.16 & & \\
\hline & & & FR & $\operatorname{cnfBd}$ & cnfisd. & cnfed. & cnfsd. \\
\hline 29 & MS 3 & WN7 & FR & $<5.5$ & $<6.2$ & $<45$. & $<130$ \\
\hline \multirow[t]{2}{*}{30} & 94305 & WC6+06-8 & $\mathbf{A}$ & 0.07 & 0.105 & $0.85:$ & $<5.0$ \\
\hline & & & FR & $<2.0$ & $<17$ & cnfsd. & cnfsd. \\
\hline $30 a$ & MS 4 & $\mathrm{WC4}+\mathrm{O4}$ & FR & $<6.7$ & $<8.6$ & $<68$ & $<95$ \\
\hline \multirow[t]{2}{*}{31} & 94546 & WN4+O8V & $\mathbf{A}$ & 0.135 & 0.075 & $<2.1$ & $<16$ \\
\hline & & & FR & $<9.1$ & $<6.4$ & $<53$ & $<320$ \\
\hline \multirow[t]{3}{*}{$31 \mathrm{a}$} & SMSP 4 & WC6 & $\mathbf{P}$ & $<0.8$ & $<1.1$ & $<23$. & $<43$ \\
\hline & & & $\mathbf{A}$ & $<0.7$ & $<0.8$ & $<4.6$ & \\
\hline & & & $\mathbf{F R}$ & $<0.9$ & $<1.8$ & $<150$ & $<340$ \\
\hline \multirow[t]{2}{*}{32} & MS 5 & WC5 & FR & $<15$ & $<28$ & $<130$ & $<410$ \\
\hline & & & A & 0.145 & 0.20 & 1.68 & \\
\hline \multirow[t]{2}{*}{33} & 95435 & WC5 & FR & $<0.6$ & $<0.6$ & $<5.7$ & $<14$ \\
\hline & & & $\mathbf{A}$ & 0.10 & 0.060 & & \\
\hline \multirow[t]{3}{*}{34} & LS 5 & WN4.5 & $\mathbf{F}$ & $<3,0$ & 2.56 & $<430$ & $<1400$ \\
\hline & & & $\mathbf{A}$ & 0.088 & 0.19 & & \\
\hline & & & FR & $<43$ & $<87$. & $<1100$ & $<1200$ \\
\hline 35 & MS 6 & WN6 & FR & $<21$. & $<81$ & $<320$ & $<750$ \\
\hline \multirow[t]{2}{*}{$35 \mathbf{a}$} & SMSP 5 & WN6 & FR & $<4.5$ & $<12$. & $<60$ & $<150$. \\
\hline & & & $\mathbf{A}$ & $<1.0$ & 0.12 & & \\
\hline \multirow[t]{2}{*}{$35 b$} & SMSP 6 & WN4 & FR & $<7.3$ & $<9.3$ & $<190$ & $<240$ \\
\hline & & & $\mathbf{A}$ & 0.030 & & & \\
\hline \multirow[t]{2}{*}{36} & LS 6 & WN4 & FR & $<7.2$ & $<7.2$ & $<70$ & $<115$ \\
\hline & & & $\mathbf{A}$ & 0.058 & 0.15 & & \\
\hline 37 & MS 7 & WN3 & FR & $<7.5$ & $<27$. & $<240$ & $<250$. \\
\hline 38 & MS 8 & WC4 & FR & $<11$. & $<25$. & $<320$ & $<380$. \\
\hline $\mathbf{3 8 a}$ & SMSP 7 & WN6 & FR & $<14$. & $<32$ & $<290$ & $<370$ \\
\hline \multirow[t]{2}{*}{$38 \mathrm{~b}$} & SMSP 8 & WC7 & FR & $<8.2$ & $<28$ & $<200$ & $<270$ \\
\hline & & & $\mathbf{A}$ & 0.125 & & & \\
\hline 39 & MS 9 & WC6 & FR & $<5.9$ & $<20$ & $<120$ & $<170$ \\
\hline 40 & 96548 & WN8(SB1) & A & 0.77 & 0.30 & $<0.48$ & $<0.12$ \\
\hline & & & $\mathbf{P}$ & 0.68 & 0.25 : & $<0.58$ & $<10.7$ \\
\hline & & & $\mathbf{F}$ & 0.69 & $<0.3$ & $<6.4$ & $<8.5$ \\
\hline & & & FR & 0.47 & 0.29 & cnfsd. & cnfsd. \\
\hline 41 & LS 7 & WC6 & FR & $<3.5$ & $<4.3$ & $<59$ & $<130$ \\
\hline & & & $\mathbf{A}$ & 0.27 & & & \\
\hline 42 & 97152 & $\mathrm{WC7}+\mathrm{O} 7 \mathrm{~V}$ & FR & $<5.0$ & $\cdots \cdots$ & $\ldots+\cdots$ & $\cdots \cdots$ \\
\hline & & & $\mathbf{A}$ & 0.28 & 0.073 & & \\
\hline $42 a$ & SMSP 9 & WN4.5 & FR & $<13$ & $<28$ & $<320$ & $<640$ \\
\hline & & & $\mathbf{A}$ & 0.10 & & & \\
\hline $42 b$ & SMSP 10 & WN3:+C & $\mathbf{F}$ & $<0.6$ & $<2.2$ & $<56$ & $<130$. \\
\hline & & & $\mathbf{A}$ & 0.131 & $<1.4$ & & \\
\hline & & & FR & $<4.9$ & $<29$ & $<120$ & $<220$ \\
\hline $42 c$ & SMSP 11 & WN6 & FR & $<92$ & $<270$ & $<3600 .<5100$ & \\
\hline $42 d$ & SMSP 12 & WN4 & FR & $<6000$. & $<39000$. & $<61000$ & $<48000$. \\
\hline 43 & 97950 & WN6+O5 & $\mathbf{F}$ & $<1100$ & $<4300$ & $<5600$ & $<19000$. \\
\hline & & & FR & $<6300$. & $<28000$ & $<27000$ & $<25000$ \\
\hline 44 & LSS 2289 & WN4 & $\mathbf{P}$ & $<0.8$ & $<0.4$ & $<2.5$ & $<22$ \\
\hline & & & A & 0.023 & & & \\
\hline & & & $\mathbf{F}$ & $<0.8$ & $<0.3$ & $<1.8$ & $<12$. \\
\hline & & & FR & $<23$. & $<4.1$ & $<8.8$ & $<13$ \\
\hline $44 a$ & SMSP 13 & WN3: & FR & $<56$. & $<190$. & $<300$ & $<530$ \\
\hline & & & A & 0.86 & 0.38 & & \\
\hline 45 & LSS 2423 & WC6 & FR & $<49$. & $<220$ & $<1100 .<1400$ & \\
\hline & & & A & 0.31 & 0.096 & & \\
\hline 46 & 104994 & WN3p & FR & $<34$. & $<14$ & $<44$. & $<85$ \\
\hline 47 & E311884 & WN6+O5V & FR & $<1.2$ & $<6.8$ & $<24$ & $<62$ \\
\hline & & & $\mathbf{A}$ & 0.19 & 0.12 & & \\
\hline $47 \mathrm{a}$ & We 21 & WN8 & $\mathbf{A}$ & 0.29 & 2.15 & 12.96 & $<69$ \\
\hline & & & FR & $<7.0$ & $<8.8$ & $<77$ & $<110$ \\
\hline 48 & 113904 & $\mathrm{WC} 6+09.5 \mathrm{I}$ & $\mathbf{A}$ & 0.50 & 0.38 & & \\
\hline
\end{tabular}


TABLE 1-Contimued

\begin{tabular}{|c|c|c|c|c|c|c|c|}
\hline$\overline{\text { WR }}$ & Name(HD) & Spectrum & $\begin{array}{l}\text { Data } \\
\text { Type }\end{array}$ & $\begin{array}{l}\mathrm{S} 12 \\
\text { [Jy] }\end{array}$ & $\begin{array}{l}\text { S25 } \\
{[\mathrm{Jy}]}\end{array}$ & $\begin{array}{l}\mathbf{S 6 0} \\
\text { [Jy] }\end{array}$ & $\begin{array}{c}\text { S100 } \\
{[\mathrm{Jy}]}\end{array}$ \\
\hline & & & $\mathbf{P}$ & 0.47 & $<0.2$ & $<1.7$ & $<16$ \\
\hline & & & $\mathbf{F}$ & 0.46 & $<1.0$ & $<0.9$ & $<5.9$ \\
\hline & & & FR & 0.25 & 0.13 & cnfsd. & cnfsd. \\
\hline $48 a$ & Danks 1 & WC8 & FR & $<95$ & $<600$ & $<1600 .<1700$. & \\
\hline \multirow[t]{2}{*}{49} & LSS 2979 & WN5 & FR & $<0.8$ & $<0.9$ & $<7.5$ & $<19$ \\
\hline & & & A & 0.20 & 0.20 & & \\
\hline \multirow[t]{3}{*}{50} & LSS 3013 & WC6+a & $\mathbf{P}$ & $<1.6$ & 1.17 & $<15$. & $<88$ \\
\hline & & & A & 0.058 & 1.05 & 5.96 & \\
\hline & & & FR & $<1.6$ & $<25$ & $<160$. & $<210$. \\
\hline \multirow[t]{2}{*}{51} & LSS 3017 & WN4 & FR & $<8.3$ & $<20$ & $<260$. & $<330$ \\
\hline & & & A & 0.24 & 0.14 & & \\
\hline \multirow[t]{3}{*}{52} & 115473 & WC5 & $\mathbf{F}$ & 0.148 & $<0.1$ & $<0.8$ & $<11$. \\
\hline & & & $\mathbf{A}$ & 0.16 & 0.18 & & \\
\hline & & & FR & 0.058 & $<0.3$ & $<1.1$ & $<1.7$ \\
\hline \multirow[t]{3}{*}{53} & 117297 & WC8 & A & 0.59 & 0.205 & & \\
\hline & & & $\mathbf{P}$ & 0.68 & $<1.5$ & $<11$. & $<98$ \\
\hline & & & FR & 0.179 & $<4.3$ & $<9.6$ & $<46$ \\
\hline 54 & LSS 3111 & WN4 & FR & $<2.6$ & $<0.9$ & $<5.5$ & $<12$ \\
\hline \multirow[t]{3}{*}{55} & 117688 & WN7 & $\mathbf{P}$ & 3.62 & 2.36 & $<11$. & $<71$ \\
\hline & & & $\mathbf{F}$ & 3.43 & 2.60 & $<56$. & $<290$. \\
\hline & & & FR & 0.385 & $<3.1$ & $<18$ & $<55$. \\
\hline 56 & LS 8 & WC7 & FR & $<0.96$ & $<3.5$ & $<3.8$ & $<24$ \\
\hline \multirow[t]{2}{*}{57} & 119078 & WC7 & A & 0.107 & 0.155 & & \\
\hline & & & FR & 0.060 & 0.029 & $<0.77$ & $<1.8$ \\
\hline 58 & LSS 3162 & WN4/WCE & FR & $<0.4$ & $<0.5$ & $<3.9$ & $<9.4$ \\
\hline 59 & LSS 3164 & WC9 & $\begin{array}{l}\text { FR } \\
\text { A }\end{array}$ & $\begin{array}{l}<18 \\
0.60\end{array}$ & $<20$ & $<190$. & $<710$ \\
\hline \multirow[t]{2}{*}{60} & 121194 & WC8 & FR & $<4.3$ & $<6.0$ & $<25$. & $<150$. \\
\hline & & & $\mathbf{A}$ & 0.42 & 0.19 & & \\
\hline \multirow[t]{2}{*}{61} & LSS 3208 & WN6 & FR & $<1.3$ & $<2.4$ & $<4.7$ & $<27$ \\
\hline & & & A & 0.023 & 0.069 & & \\
\hline \multirow[t]{2}{*}{62} & NS 2 & WN6 & FR & $<6.0$ & $<6.2$ & $<32$. & $<130$. \\
\hline & & & A & 0.30 & 0.089 & & \\
\hline 63 & LSS 3289 & WN6 & $\begin{array}{l}\text { FR } \\
\text { A }\end{array}$ & $\begin{array}{l}<23 . \\
0.56\end{array}$ & $\begin{array}{l}<45 \\
0.36\end{array}$ & $<380$ & $<860$. \\
\hline \multirow[t]{2}{*}{64} & BS 3 & WC7 & FR & $<1.5$ & $<0.8$ & $<4.3$ & $<22$. \\
\hline & & & A & 0.17 & 0.049 & & \\
\hline 65 & LSS 3319 & WC9 & FR & $<38$ & $<33$ & $<250$ & $<660$. \\
\hline 66 & 134877 & WN8 & FR & $<6.4$ & $<2.7$ & $<75$ & $<140$. \\
\hline & & & A & 0.064 & 0.083 & 0.29 & \\
\hline 67 & LSS 332y & WN6 & FR & $<4.8$ & $<5.7$ & $<49$. & $\ldots \ldots$. \\
\hline & & & $\mathbf{A}$ & 0.20 & & & \\
\hline 68 & BS 4 & WC7 & FR & $<7.1$ & $<11$. & $<70$. & $<160$. \\
\hline & & & $\mathbf{A}$ & 0.068 & 0.113 & & \\
\hline 69 & 136488 & WC9 & A & 1.02 & 0.305 & 0.71 & \\
\hline & & & $\mathbf{P}$ & 0.89 & 0.20 & $<0.6$ & $<26$. \\
\hline & & & $\mathbf{F}$ & 1.03 & 0.32 & $<1.7$ & $<34$ \\
\hline & & & FR & 1.24 & 0.53 & 0.52 & $<11$. \\
\hline 70 & 137603 & WC9+BoI & A & 6.54 & 2.09 & 0.69 & $<13$ \\
\hline & & & $\mathbf{P}$ & 6.53 & 2.01 & $<11$ & $<120$. \\
\hline & & & $\mathbf{F}$ & 6.51 & 2.01 & $<3.8$ & $<30$ \\
\hline & & & FR & 5.25 & 1.46 & 0.99 & $<14$. \\
\hline 71 & 143414 & WN6(SB1) & $\mathbf{P}$ & $<0.9$ & $<0.2$ & 0.38 & 3.98: \\
\hline & & & $\mathbf{A}$ & 0.072 & 0.073 & & \\
\hline & & & $\mathbf{F}$ & $<0.1$ & $<0.08$ & 0.78 & $<7.4$ \\
\hline & & & FR & $<0.2$ & $<0.2$ & $<2.1$ & cnfsd. \\
\hline 73 & NS 3 & WC9 & A & 0.56 & 0.23 & 1.05 & 3.55 \\
\hline & & & $P$ & 0.50 & $<0.3$ & $<1.5$ & $<29$. \\
\hline & & & $\mathrm{F}$ & 0.54 & $<0.4$ & $<2.9$ & $<12$ \\
\hline & & & FR & 0.440 & 0.142 & 0.826 & $<9.6$ \\
\hline 74 & BP 1 & WN7 & A & & $<107$ & $<80$ & $<34$ \\
\hline & & & $\mathbf{P}$ & $<155$ & $<110$ & $<40$. & $<390$. \\
\hline & & & F & $<159$ & $<110$. & $<84$ & $<73$ \\
\hline & & & FR & $<160$. & $<26$ & $<240$. & $<260$. \\
\hline 75 & 147419 & WN5 & FR & $<9.8$ & $<24$ & $<180$ & $<220$. \\
\hline
\end{tabular}


TABLE 1-Continued

\begin{tabular}{|c|c|c|c|c|c|c|c|}
\hline$\overline{\overline{W R}}$ & Name(HD) & $\overline{\text { Spectrum }}$ & $\begin{array}{l}\text { Data } \\
\text { Type }\end{array}$ & $\begin{array}{l}\mathrm{S12} \\
{[\mathrm{Jy}]}\end{array}$ & $\begin{array}{l}\mathrm{S25} \\
{[\mathrm{Jy}]}\end{array}$ & $\begin{array}{l}\mathbf{S 6 0} \\
\text { [Jy] }\end{array}$ & $\begin{array}{l}\mathbf{S 1 0 0} \\
{[\mathrm{Jy}]}\end{array}$ \\
\hline \multirow[t]{3}{*}{76} & LSS 3693 & WC9 & $\overline{\mathbf{P}}$ & 16.75 & 34.7: & $<2600$ & $<6800$ \\
\hline & & & A & 16.02 & 22.83 & 66.65: & \\
\hline & & & FR & $<260$ & $<2200$ & cnfsd. & cnfsd. \\
\hline \multirow[t]{2}{*}{77} & He3-1239 & WC8 $(+a)$ & $P$ & $<2.8$ & $<2.7$ & $<6.0$ & $<150$. \\
\hline & & & $\mathbf{F R}$ & $<6.8$ & $<10$ & $<70$ & $<150$ \\
\hline \multirow[t]{3}{*}{78} & 151932 & WN7 & A & 1.29 & 0.57 & & \\
\hline & & & $\mathbf{P}$ & 1.30 & 0.59 & $<4.9$ & $<43$ \\
\hline & & & FR & $<1.3$ & $<5.8$ & $<16$ & $<94$. \\
\hline \multirow[t]{2}{*}{79} & 152270 & $\mathrm{WC} 7+\mathrm{O} 5-8$ & A & 0.69 & 0.415 & & \\
\hline & & & FR & $<0.5$ & $<2.7$ & $<14$ & $<42$ \\
\hline \multirow[t]{4}{*}{80} & LSS 3871 & WC9 & A & 1.88 & 0.47 & & \\
\hline & & & $\mathrm{P}$ & 1.88 & 0.58 : & $<13$ & $<130$ \\
\hline & & & $\mathbf{F}$ & 1.67 & $<1.5$ & $<36$ & $<400$ \\
\hline & & & FR & 1.564 & 0.280 & $<65$ & $<58$ \\
\hline \multirow[t]{2}{*}{81} & He3-1316 & WC9 & FR & 0.462 & 0.305 & $<28$ & $<65$ \\
\hline & & & A & 0.33 & 0.17 & & \\
\hline \multirow[t]{2}{*}{82} & LS 11 & WN8 & A & 0.25 & 0.23 & & \\
\hline & & & FR & 0.156 & 0.095 & $<12$ & $<59$ \\
\hline \multirow[t]{2}{*}{83} & He3-1344 & WN6 & FR & $<2.8$ & $<2.5$ & $<12$ & $<53$ \\
\hline & & & $\mathbf{A}$ & 0.080 & 0.092 & 0.15 & \\
\hline \multirow[t]{2}{*}{84} & The 3 & WN6 & FR & $<21$ & $<26$ & $<280$. & $<540$ \\
\hline & & & A & 0.27 & & & \\
\hline \multirow[t]{4}{*}{85} & LSS 3982 & WN6 & $\mathbf{A}$ & $<71$. & $<52$. & $<8.4$ & \\
\hline & & & $\mathbf{P}$ & $<68$. & $<50$ & $<6.2$ & $<470$ \\
\hline & & & $\mathbf{F}$ & $<62$. & $<49$ & $<34$ & $<91$. \\
\hline & & & FR & $<55$ & $<38$ & $<17$ & $<100$ \\
\hline \multirow[t]{2}{*}{86} & 156327 & WC7+a & $\mathbf{A}$ & 0.26 & 0.215 & 1.60 & \\
\hline & & & $\mathbf{F R}$ & $<1.2$ & $<1.4$ & $<6.2$ & $<31$ \\
\hline \multirow[t]{2}{*}{87} & LSS 4064 & WN7 & A & 0.77 & 3.72 & 14.17 & \\
\hline & & & FR & $<2.5$ & $<36$ & $\ldots \ldots$. & $<20$ \\
\hline 88 & The 1 & WC9 & FR & 2.58 & $<47$. & $<220$ & $<170$ \\
\hline & & & $\mathbf{A}$ & 1.17 & & & \\
\hline 89 & LSS 4065 & WN7 & A & 0.39 & 3.17 & 21.59 & \\
\hline & & & $\mathbf{P}$ & $<3.3$ & 3.49 & $<50$ & $<260$ \\
\hline & & & FR & $<2.0$ & $<88$ & $<200$ & $<460$ \\
\hline 90 & 156385 & WC7 & $\mathbf{P}$ & 0.95 & 0.47 & $<0.6$ & $<6.2$ \\
\hline & & & A & 0.97 & 0.28 & 0.30 & \\
\hline & & & $\mathbf{F}$ & 0.81 & 0.40 & $<1.0$ & $<8.0$ \\
\hline & & & FR & 0.885 & 0.390 & $<5.1$ & $<14$ \\
\hline 91 & StSa 1 & WN7 & FR & $<2500$ & $<14000$ & $<44000$ & $<34000$ \\
\hline 92 & 157451 & WC9 & A & 0.06 & 0.18 & $<1.0$ & \\
\hline & & & FR & $<0.32$ & $<0.43$ & $<3.5$ & $<5.1$ \\
\hline 93 & 157504 & WC7+O7-9 & FR & $<8200$ & cnfsd. & cnfsd. & cnfsd. \\
\hline $93 a$ & PKS359+3.1 & WN2.5-3 & FR & $<1.4$ & $<5.0$ & $<1.0$ & $<9.9$ \\
\hline & & & $A$ & 0.055 & 0.10 & & \\
\hline 94 & 158860 & WN6 & FR & $<5.7$ & $<6.5$ & $<100$ & $<190$. \\
\hline & & & A & 0.31 & & & \\
\hline 95 & He3-1434 & WC9 & $\mathbf{P}$ & 4.56 & $<1.7$ & $<12$ & $<200$. \\
\hline & & & $\mathbf{A}$ & 4.02 & 3.39 & 2.92 & \\
\hline & & & FR & $<41$. & $<23$ & $<49$ & $<120$ \\
\hline 96 & LSS 4265 & WC9 & $\mathbf{P}$ & 1.73 & $<3.4$ & $<27$ & $<430$ \\
\hline & & & $\mathbf{A}$ & 1.61 & 0.66 & & \\
\hline & & & $\mathbf{F}$ & $<1.9$ & $<4.3$ & $<32$ & $<150$. \\
\hline & & & FR & 0.436 & $<1.2$ & $<110$ & $<360$ \\
\hline 97 & E320102 & WN3+O5-7 & $\mathbf{P}$ & $<1.0$ & $<1.9$ & $<1.8$ & $<97$ \\
\hline & & & A & 0.089 & 0.21 & 1.38 & \\
\hline & & & FR & $<0.7$ & $<0.8$ & $<9.3$ & $<30$ \\
\hline 98 & E318016 & WC7/WN6 & FR & $<2.5$ & $<1.6$ & $<44$ & $<120$ \\
\hline & & & $\mathbf{A}$ & 0.54 & & & \\
\hline $98 \mathbf{a}$ & IRAS17380 & WC9 & $\mathbf{A}$ & 47.84 & 13.97 & & \\
\hline & & & $\mathbf{P}$ & 51.63 & 14.10 & $<22$. & $<410$ \\
\hline & & & $\mathbf{F}$ & 53.83 & 13.83 & $<100$ & $<1100$ \\
\hline & & & FR & 31.69 & 11.13 & 18.91 & $<810$ \\
\hline 100 & E318139 & WN6 & $\mathrm{P}$ & 4.22 & 11.08: & 95.32 & $<96$. \\
\hline & & & A & 0.42 & 0.22 & 0.39 & \\
\hline & & & FR & $<1.7$ & $<1.3$ & $<6.9$ & $<17$ \\
\hline
\end{tabular}


TABLE 1-Continued

\begin{tabular}{|c|c|c|c|c|c|c|c|}
\hline$\overline{\mathrm{WR}}$ & Name(HD) & Spectrum & $\begin{array}{l}\text { Data } \\
\text { Type }\end{array}$ & $\begin{array}{l}\text { S12 } \\
{[\mathrm{Jy}]}\end{array}$ & $\begin{array}{l}\overline{\mathrm{S25}} \\
{[\mathrm{Jy}]}\end{array}$ & $\begin{array}{l}\mathrm{S} 60 \\
{[\mathrm{Jy}]}\end{array}$ & $\begin{array}{l}\mathbf{S 1 0 0} \\
{[\mathrm{Jy}]}\end{array}$ \\
\hline \multirow[t]{3}{*}{101} & DA 3 & WC8 & $\mathbf{P}$ & $<5.0$ & $<7.0$ & 37.61 & $<110$ \\
\hline & & & F & $<5.2$ & $<13$ & $<81$. & $<340$ \\
\hline & & & FR & $<14$. & $<38$. & $<150$. & $<90$. \\
\hline 102 & Sand 4 & WO1 & $\begin{array}{l}\text { F } \\
\text { FR }\end{array}$ & $\begin{array}{l}<3.0 \\
\text { cnfsd. }\end{array}$ & $\begin{array}{c}8.92 \\
\text { cnfsd. }\end{array}$ & $\begin{array}{c}32.56 \\
\text { cnfsd. }\end{array}$ & $\begin{array}{l}<4800 \text {. } \\
\text { cnfsd. }\end{array}$ \\
\hline \multirow[t]{4}{*}{103} & 164270 & WC9(SB1?) & A & 1.06 & 0.36 & $<0.9$ & \\
\hline & & & $\mathbf{P}$ & 1.03 & $<1.6$ & $<3.3$ & $<32$ \\
\hline & & & $\mathrm{F}$ & 0.88 & $<0.7$ & $<1.3$ & $<10$. \\
\hline & & & FR & 0.801 & 0.183 & $<1.8$ & $<9.0$ \\
\hline \multirow[t]{4}{*}{104} & $\mathrm{Ve} 2-45$ & WC9 & $\mathbf{A}$ & 412.3 & 133.0 & 19.94 & \\
\hline & & & $\mathbf{P}$ & 411.5 & 140.2 & $<38$ & $<330$ \\
\hline & & & $\mathrm{F}$ & 398.8 & 126.1 & $<140$. & $<1300$. \\
\hline & & & FR & 394.21 & 169.37 & $<450$ & $<390$. \\
\hline \multirow[t]{2}{*}{105} & NS $4, \mathrm{Ve} 2-47$ & WN8 & A & 1.80 & 1.32 & $<7.1$ & \\
\hline & & & FR & $<15$. & $<27$ & $<140$. & $<170$. \\
\hline \multirow[t]{4}{*}{106} & E313643 & WC9 & A & 12.04 & 3.97 & 14.83 & $<100$ \\
\hline & & & $\mathbf{P}$ & 11.67 & 3.76 & 11.69: & $<400$ \\
\hline & & & $\mathrm{F}$ & 11.20 & $<8.5$ & $<81$ & $<440$ \\
\hline & & & FR & 10.22 & 4.26 & 20.07 & $<310$ \\
\hline \multirow[t]{2}{*}{107} & DA 1 & WN8 & FR & $<6.2$ & $<74$ & cnfsd. & cnfsd. \\
\hline & & & $\mathbf{A}$ & 2.73 & & & \\
\hline \multirow[t]{2}{*}{108} & E313846 & WN9 & FR & $<10$ & $<5.4$ & $<48$ & $<210$ \\
\hline & & & A & 0.37 & 0.14 & & \\
\hline \multirow[t]{2}{*}{109} & NS 5 & WN3 & $P$ & $<0.2$ & $<0.3$ & $<0.4$ & $<6.6$ \\
\hline & & & FR & $<0.5$ & $<0.8$ & $<2.9$ & $<5.1$ \\
\hline 110 & 165688 & WN6 & FR & $\begin{array}{l}<17 . \\
0.39\end{array}$ & $\begin{array}{l}<15 . \\
0.47\end{array}$ & $<200$ & $<650$. \\
\hline \multirow[t]{2}{*}{111} & 165763 & WC5 & A & 0.79 & 0.47 & $<19$. & $<37$. \\
\hline & & & FR & $<5.3$ & $<3.6$ & $<17$ & $<450$. \\
\hline \multirow[t]{4}{*}{112} & GL 2104 & WC9 & $\mathbf{A}$ & 159.36 & 74.59 & 20.86 & $<18$ \\
\hline & & & $\mathbf{P}$ & 150.4 & 74.04 & 19.33 & $<320$ \\
\hline & & & $\mathrm{F}$ & 156.4 & 72.48 & $<42$ & $<180$ \\
\hline & & & FR & 132.76 & 69.48 & 12.53 & 7.33 \\
\hline \multirow[t]{3}{*}{113} & 168206 & WC8+O8-9IV & $\mathbf{P}$ & 2.17 & 2.82: & $<120$. & $<630$. \\
\hline & & & A & 2.63 & 1.78 & & \\
\hline & & & FR & $<18$. & $<45$. & cnfsd. & cnfsd. \\
\hline 114 & 169010 & WC5 & $\mathbf{P}$ & $<500$ & $<330$ & $<73$ & $<260$ \\
\hline & & & $F$ & $<480$ & $<310$ & $<63$ & $<750$. \\
\hline & & & FR & $<34$ & $<25$ & $<91$ & $<120$ \\
\hline 115 & IC14-19 & WN6 & A & 4.82 & 4.19 & 2.81 & \\
\hline & & & $\mathbf{P}$ & $<7.7$ & $<4.6$ & $<16$ & $<250$ \\
\hline & & & FR & 4.35 & 3.02 & $<9.8$ & $<16$ \\
\hline 116 & ST 1 & WN8 & $P$ & $<3.8$ & 8.57 & $<37$ & $<190$ \\
\hline & & & $\mathbf{A}$ & 0.80 & 7.97 & & \\
\hline & & & $\mathrm{F}$ & $<3.1$ & 6.94 & $<840$. & $<9400$. \\
\hline & & & FR & $<21$. & $<75$. & $<380$ & $<1000$. \\
\hline 117 & IC $14-22$ & WC8 & $\mathrm{P}$ & $<2.8$ & $<1.3$ & $<5.1$ & $<54$ \\
\hline & & & $\mathbf{F}$ & $<2.8$ & $<1.3$ & $<5.2$ & $<30$. \\
\hline & & & FR & 0.282 & 0.134 & $<10$ & $<16$. \\
\hline 118 & GL 2179 & WC9 & A & 72.29 & 21.62 & 35.29 & 105.25 \\
\hline & & & $\mathbf{P}$ & 70.36 & 21.69 & $<37$ & $<380$ \\
\hline & & & F & 69.82 & 21.25 & $<140$. & $<1000$. \\
\hline & & & FR & 65.15 & 24.33 & 31.78 & $<490$ \\
\hline 119 & The 2 & WC9 & FR & 1.44 & $<7.4$ & $<32$ & $<115$ \\
\hline & & & $\mathbf{A}$ & 0.41 & 0.22 & & \\
\hline 120 & Vyl-3 & WN7 & FR & $<8.8$ & $<11$. & $<110$. & $<640$ \\
\hline & & & A & 0.27 & & & \\
\hline 121 & AS 320 & WC9 & A & 2.82 & 1.38 & 1.77: & \\
\hline & & & FR & 2.27 & 1.26 & $<10$. & $<260$ \\
\hline 123 & 177230 & WN8(SB1) & FR & $<0.3$ & $<0.2$ & $<1.5$ & $<3.0$ \\
\hline & & & A & 0.090 & 0.071 & & \\
\hline 125 & IC14-36 & WC7 & F & 0.85 & $<3.5$ & $<210$. & $<960$. \\
\hline & & & A & 1.11 & 1.48 & & \\
\hline & & & FR & $<44$. & $<61$. & cnfsd. & cnfsd. \\
\hline 126 & ST 2 & WC5/WN & $\mathbf{P}$ & $<0.9$ & $<0.7$ & $<1.4$ & $<7.0$ \\
\hline & & & A & 0.065 & 0.035 & & \\
\hline
\end{tabular}


TABLE 1-Comtinued

\begin{tabular}{|c|c|c|c|c|c|c|c|}
\hline$\overline{\text { WR }}$ & Name(HD) & Spectrum & $\begin{array}{l}\text { Data } \\
\text { Type }\end{array}$ & $\begin{array}{l}\text { S12 } \\
\text { [Jy] }\end{array}$ & $\begin{array}{l}\text { S25 } \\
\text { [Jy] }\end{array}$ & $\begin{array}{l}\mathrm{S} 60 \\
{[\mathrm{Jy}]}\end{array}$ & $\begin{array}{l}5100 \\
{[\mathrm{Jy}]}\end{array}$ \\
\hline & & & $F$ & $<0.4$ & $<0.2$ & $<2.6$ & $<13$ \\
\hline & & & FR & $<1.5$ & $<1.8$ & $<4.5$ & $<9.7$ \\
\hline 127 & 186943 & WN4+09.5V & $\begin{array}{l}\text { FR } \\
\text { A }\end{array}$ & $\begin{array}{c}<1.2 \\
0.041\end{array}$ & $<3.6$ & $<26$ & $<27$ \\
\hline \multirow[t]{2}{*}{128} & 187282 & WN4 (SB1) & $A$ & 0.040 & 0.045 & 0.125 & \\
\hline & & & FR & 0.023 & $<0.3$ & $<2.9$ & $<3.0$ \\
\hline \multirow[t]{4}{*}{130} & LS 16 & WN8 & $\mathbf{P}$ & 1.55 & 13.16 & 48.22 & $<50$ \\
\hline & & & $\mathbf{A}$ & 1.31 & 11.70 & 36.49 & 29.78 \\
\hline & & & $\mathbf{F}$ & 1.66 & 12.88 & 38.75 & $<66$ \\
\hline & & & FR & 1.85 & 9.44 & 31.74 & 47.33 \\
\hline \multirow[t]{3}{*}{131} & IC14-52 & WN7+a & $\mathbf{P}$ & $<0.6$ & $<2.2$ & $<36$ & $<200$ \\
\hline & & & $\mathrm{F}$ & $<1.7$ & $<2.3$ & $<160$. & $<800$ \\
\hline & & & FR & $<2.9$ & $<7.6$ & $<54$ & $<100$ \\
\hline \multirow[t]{2}{*}{132} & 190002 & WC6 & $\mathbf{P}$ & $<0.6$ & $<0.2$ & $<4.8$ & $<54$ \\
\hline & & & FR & $<1.5$ & $<0.6$ & $<3.7$ & $<17$. \\
\hline \multirow[t]{3}{*}{133} & 190918 & WN4.5+09.5Ib & A & 0.345 & 0.14 & 0.42 & $<6.4$ \\
\hline & & & $\mathbf{P}$ & 0.34 & $<0.4$ & $<6.8$ & $<59$ \\
\hline & & & FR & 0.196 & 0.028 & $<4.8$ & $<8.8$ \\
\hline \multirow{3}{*}{134} & 191765 & WN6(SB1) & A & 0.62 & 0.39 & 0.35 & $<1.0$ \\
\hline & & & $\mathrm{P}$ & 0.67 & $0.31:$ & $<14$ & $<49$ \\
\hline & & & FR & 0.679 & 0.378 & $<6.4$ & $<6.8$ \\
\hline \multirow{2}{*}{135} & 192103 & WC8 & A & 0.24 & 0.19 & 0.31 & \\
\hline & & & FR & 0.141 & 0.090 & $<18$ & $<55$ \\
\hline \multirow[t]{4}{*}{136} & 192163 & WN6(SB1) & $\mathbf{P}$ & 1.33 & 0.58 & $<6.4$ & $<61$ \\
\hline & & & A & 1.36 & 0.51 & & \\
\hline & & & $\mathbf{F}$ & 1.33 & $<1.3$ & $<19$ & $<87$ \\
\hline & & & FR & 1.269 & 0.561 & $<130$ & $<51$ \\
\hline \multirow[t]{3}{*}{137} & 192641 & $\mathrm{WC} 7+\mathrm{OB}$ & A & 2.93 & 2.21 & 16.60 & 64.73 \\
\hline & & & $\mathbf{P}$ & 3.21 & 2.37: & 26.8: & $<200$ \\
\hline & & & FR & 3.564 & 3.378 & $<57$ & $<75$ \\
\hline \multirow[t]{3}{*}{138} & 193077 & WN6+O9 & A & 0.36 & 0.225 & 0.12 & $<5.2$ \\
\hline & & & $\mathbf{P}$ & 0.43 & $<0.25$ & $<11$. & $<130$ \\
\hline & & & FR & 0.526 & $<0.23$ & $<22$. & $<70$ \\
\hline \multirow[t]{3}{*}{139} & 193576 & WN5+O6 & A & 0.59 & 0.26 & 0.11 & \\
\hline & & & $\mathbf{P}$ & 0.57 & $<0.48$ & $<18$ & $<210$. \\
\hline & & & FR & 0.654 & 0.476 & $<30$ & $<39$ \\
\hline \multirow[t]{4}{*}{140} & 193793 & WC7 +04-5 & $\mathbf{A}$ & 1.91 & 1.04 & $<1.2$ & \\
\hline & & & $\mathbf{P}$ & 1.89 & 1.11 & $<21$. & $<210$ \\
\hline & & & $\mathbf{F}$ & 1.81 & 0.94 & $<12$ & $<46$ \\
\hline & & & FR & 1.154 & 0.598 & $<23$ & $<110$ \\
\hline 141 & 193928 & WN6(SB1) & $\begin{array}{l}\mathrm{P} \\
\text { FR }\end{array}$ & $\begin{array}{l}<840 . \\
\text { cnfsd. }\end{array}$ & $\begin{array}{l}<240 . \\
\text { cnfsd. }\end{array}$ & $\begin{array}{c}<51 \\
\text { cnfsd. }\end{array}$ & $\begin{array}{c}<93 \\
\text { cnfsd. }\end{array}$ \\
\hline 142 & ST 3 & WO2 & FR & $<22$ & $<24$ & $<51$ & $<730$ \\
\hline 143 & 195177 & WC5 & A & 0.29 & 0.32 & 3.38 & $<43$ \\
\hline & & & FR & $<5.2$ & $<6.7$ & $<62$. & $<74$ \\
\hline 144 & HM19-1 & WC4 & FR & $<7.4$ & $<28$ & $<260$ & $<320$ \\
\hline & & & A & 0.37 & 0.38 & & \\
\hline 145 & AS 422 & WN3/WCE(SB1) & $\mathbf{P}$ & 0.61 & $<2.7$ & $<42$ & $<330$. \\
\hline & & & $\mathbf{A}$ & 0.55 & 0.22 & 0.23: & \\
\hline & & & FR & $<0.32$ & $<29$ & $<250$ & $<220$ \\
\hline 146 & HM19-3 & WC6 & $\mathbf{P}$ & 0.97 & $<2.2$ & $<20$ & $<190$ \\
\hline & & & A & 0.84 & & & \\
\hline & & & FR & 1.218 & $<17$ & $<69$ & $<18$ \\
\hline 147 & NS 6 & WN8 & A & 5.40 & 3.03 & 1.35 & \\
\hline & & & $\mathrm{P}$ & 5.30 & 3.12 & $<19$ & $<210$. \\
\hline & & & $F$ & 5.14 & $<5.9$ & $<7.0$ & $<110$. \\
\hline & & & FR & 3.73: & 1.46: & $<94$ & $<130$. \\
\hline 148 & 197406 & WN7(SB1) & FR & $<0.4$ & $<0.5$ & $<1.8$ & $<4.2$ \\
\hline & & & A & 0.11 & $0.05:$ & & \\
\hline 149 & ST 4 & WN6-7 & FR & $<0.8$ & $<0.7$ & $<2.0$ & $<10$ \\
\hline & & & A & 0.080 & 0.067 & & \\
\hline 150 & ST 5 & WC5 & A & 0.075 & 0.060 & 0.30 & 3.40 \\
\hline & & & FR & $<1.0$ & $<0.9$ & $<4.1$ & $<10$ \\
\hline 151 & CX Cep & WN4+O8V & FR & $<0.8$ & $<1.1$ & $<2.7$ & $<9.6$ \\
\hline & & & $\mathrm{A}$ & 0.095 & 0.033 & & \\
\hline 152 & 211564 & WN3 & $\mathrm{FR}$ & $<2.8$ & $<3.5$ & $<27$. & $<58$ \\
\hline
\end{tabular}


TABLE 1-Cimimued

\begin{tabular}{ccllllll}
\hline \hline WR & Name(HD) & Spectrum & $\begin{array}{l}\text { Data } \\
\text { Type }\end{array}$ & $\begin{array}{c}\text { S12 } \\
{[\mathrm{Jy}]}\end{array}$ & $\begin{array}{c}\text { S25 } \\
{[\mathrm{Jy}]}\end{array}$ & $\begin{array}{c}\text { S60 } \\
{[\mathrm{Jy}]}\end{array}$ & $\begin{array}{c}\text { S100 } \\
{[\mathrm{Jy}]}\end{array}$ \\
\hline 153 & 211853 & WN6/WCE+O & FR & $<29$. & $<75$ & $<440$. & $<600$. \\
& & & A & 0.25 & & & \\
154 & 213049 & WC6 & FR & $<0.5$ & $<0.6$ & $<2.1$ & $<6.8$ \\
& & & A & 0.10 & 0.036 & & \\
155 & 214419 & WN7+O & A & & 0.18 & 0.09 & $<0.9$ \\
& & & FR & 0.058 & 0.054 & $<3.5$ & $<6.9$ \\
156 & \multirow{2}{*}{ AC+60 38562 } & WN8 & A & 0.335 & 0.27 & 0.41 & 0.33 \\
& & & FR & 0.301 & 0.204 & $<8.8$ & $<28$. \\
157 & \multirow{2}{*}{219460} & WN4.5(+B) & FR & $<3.6$ & $<3.3$ & $<49$. & $<75$. \\
& & & A & 0.17 & 0.094 & & \\
158 & \multirow{2}{*}{ AS 513 } & WN7 & A & 0.15 & 0.11 & 0.125 & \\
& & & FR & 0.308 & $<2.3$ & $<1.2$ & cnfsd. \\
\hline
\end{tabular}

formation was quantitatively valuable in order to evaluate meaningful limits in the absence of detections. In such cases, I evaluated the standard deviation of the emission within an appropriately sized and oriented elliptical aperture at the W-R star's location, and the standard deviation of the sky background emission assessed from between three and 10 locations around the W-R. I then took the root-sum-square (RSS) of the two independent deviations and estimated the upper limit at 3 times this RSS value. A reevaluation of all matches was made in light of clear discrepancies between the different methods of measurement, and particular scrutiny was given to alleged matches with large apparent spatial offsets (greater than $\left.40^{\prime \prime}-60^{\prime \prime}\right)$.

Notes on two individual stars are in order. I omitted WR 124 because of the intimate association of this WN star with the nebula, M1-67 (Cohen \& Barlow 1975; van der Hucht et al. 1985b). AS 320 is the fainter companion of a bright IRAS source. Both the PSC and the FSS tabulate the sum of the two objects. ADDSCAN and FRESCO are capable of resolving the objects. Data in Table 1 represent direct measurements of AS 320 with ADDSCAN and FRESCO (the latter at $12 \mu \mathrm{m}$ only), and independent indirect estimates with FRESCO (subtracting the brighter source's flux densities from the sum of the two sources).

Table 2 represents the weighted combination of all these separate flux estimates, for all the W-R stars, with weights of 3 for $F$ and $P, 2$ for $A$, and 1 for FR. If a colon appears after a flux density in Table 1, then weights of $1.5,1$, or 0.5 were used for F or P, A, and FR, respectively. FRESCO's principal value was in deciding how confused a region is, and offering some insight into the P, F, and especially A flux densities, rather than in providing accurate photometry itself. When the background confusion is so high that no meaningful quantitative upper limits can be obtained even with FRESCO, the entry "cnfsd." appears in Tables $I$ and 2 . In many cases the background may vary so drastically that no useful upper limits can be obtained with an ADDSCAN; in such situations a blank appears in the line. Table 2 is my final IRAS archive on the W-R stars themselves, as opposed to their environs.

\section{COLOR-COLOR SEPARATIONS}

Using standard hot color-corrected IRAS magnitudes (e.g., see Wainscoat et al. $1992, \S 1$ ), the flux densities were con- verted into observed magnitudes and hence colors. Before exploring the potential dependence of $I R A S$ colors on W-R subclass, we note the unifying approach of Morris et al. (1993), in which it was found that many W-R stars without circumstellar dust emission show a single power-law spectrum from ultraviolet to near-infrared wavelengths, independent of both WN and WC type, and of subclass within these types. These authors felt that these power laws, whose slopes appear to be dependent on mass-loss rate but not on atmospheric abundances, could be extended directly into the mid-infrared on the basis of $I R A S$ flux densities reported for three WN stars by Mathis et al. (1992), whose primary interest was in the environs of these W-R stars rather than in the stars themselves. Morris et al. (1993) focused their attention on single $W-R$ stars because of uncertainties in the UV arising from the lack of detailed knowledge of the precise character of the companions. It is, therefore, worthwhile to investigate these authors' hypothesis before proceeding further in the analysis of IRAS colors. Specifically, is there $I R 4 S$ evidence for any statistically significant differences between nondusty single and binary W-R stars? (The presence of a hot circumstellar dust shell completely overwhelms any other sources of radiation in W-R stars, whether single or binary.)

Initially, I restrict attention to those W-R stars studied by Morris et al. (1993) and assigned to the "single" or "binary" category by them because information on the power-law spectral character currently exists solely for these stars. Table 3 , therefore, summarizes my IRAS color data for all nondusty W-R stars, separating these into WN or WC classes and into the single or binary category before recombining them into two samples corresponding to "all-single" and "all-binary" W-R stars. These restricted samples are smaller than the set of W-R stars for which IRAS provides useful data; nonetheless, they are meaningful, except for the tiny samples of stars with [60] - [100] colors that I have omitted. I detect no statistically significant ( $\geq 3 \sigma$ ) differences between single (as defined by Morris et al. 1993) and binary (also so defined) W-R stars in either [12] - [25] or [25]-[60]; i.e., the (mean $\pm 3 \sigma$ ) color ranges of single and binary $W-R$ stars are not distinct. In spite of the absence of any significant distinction, the relevance of binarity is still worth investigating with the larger sample. (One also sees a direct conflict between several stars designated as "single" by Morris et al. yet as "binary" by HHASD, suggesting the merit of a reanalysis.) Table 3 , therefore, includes the 
TABLE 2

COMBINEd IRAS PHOTOMETry fRom All DATA SETS

\begin{tabular}{|c|c|c|c|c|c|c|}
\hline$\overline{\text { WR }}$ & Name(HD) & Spectrum & $\begin{array}{l}\mathrm{S} 12 \\
{[\mathrm{Jy}]}\end{array}$ & $\begin{array}{l}\text { S25 } \\
\text { [Jy] }\end{array}$ & $\begin{array}{l}\mathrm{S} 60 \\
{[\mathrm{Jy}]}\end{array}$ & $\begin{array}{l}\mathbf{S 1 0 0} \\
{[\mathrm{Jy}]}\end{array}$ \\
\hline 1 & 4004 & WN5 & 0.25 & 0.15 & $<0.5$ & $<3.0$ \\
\hline 2 & 6327 & WN2 & 0.040 & 0.077 & 0.17 & $<8.3$ \\
\hline 3 & 9974 & WN3+a(SB1) & 0.027 & 0.037 & 0.072 & $<0.3$ \\
\hline 4 & 16523 & WC5 (SB1) & 0.10 & 0.064 & 0.039 & $<1.2$ \\
\hline 5 & 17638 & WC6 & 0.18 & 0.064 & $<0.3$ & $<1.5$ \\
\hline 6 & 50896 & WN5 (SB1) & 1.03 & 0.60 & 0.78 & $<1.8$ \\
\hline 7 & 56925 & WN4 & 0.11 & 0.47 & 9.45 & 16.9: \\
\hline 8 & 62910 & WC4/WN6 & 0.12 & 0.19 & 0.99 & $<2.5$ \\
\hline 9 & 63099 & WC5+O7 & 0.14 & 0.069 & 0.13 & $<0.9$ \\
\hline 10 & 65865 & WN4.5 & 0.11 & 0.133 & 0.12 & cnfsd. \\
\hline 11 & 68273 & WC8+O9I & 19.38 & 8.52 & 4.24 & 3.07 \\
\hline 12 & CD-45 4482 & WN7 (SB1) & 0.14 & 0.10 & cnfsd. & cnfsd. \\
\hline 13 & Ve 6-15 & WC6 & 0.072 & 0.059 & cnfsd. & cnfsd. \\
\hline 14 & 76536 & WC6 & $<5.2$ & $<8.7$ & $<2.9$ & $<21$ \\
\hline 15 & 79573 & WC6 & 0.62 & 0.34 & 1.60 & $<21$ \\
\hline 16 & 86161 & WN8 & 0.48 & 0.28 & $<1.3$ & $<26$. \\
\hline 17 & 88500 & WC5 & 0.047 & 0.053 & $<0.6$ & $<1.2$ \\
\hline 18 & 89358 & WN5 & 0.43 & 0.27 & ...... & $<130$ \\
\hline 19 & LS 3 & WC4 & 0.27 & 0.34 & $<140$ & $<280$ \\
\hline $19 a$ & SMSP 1 & WN7 & $<9.8$ & $<9.3$ & $<160$ & $<300$ \\
\hline 20 & BS 1 & WN4.5 & 0.076 & 0.079 & 0.42 & 1.00 \\
\hline $20 a$ & SMSP 2 & WN7 & cnfsd. & cnfsd. & cnfsd. & cnfsd. \\
\hline $20 \mathrm{~b}$ & SMSP 3 & WN7 & cnfsd. & cnfsd. & $\ldots \ldots$ & $\ldots . .$. \\
\hline 21 & 90657 & WN4+O4-6 & 0.27 & $<2.2$ & $<3.4$ & $<26$ \\
\hline 22 & 92740 & WN7+a(SB1) & 1.80 & $<8.7$ & $<100$ & $<770$ \\
\hline 23 & 92809 & WC6 & $<28$ & $<36$ & $<400$. & $<1300$ \\
\hline 24 & 93131 & WN7+a & $<210$ & $<1800$ & $<5900$ & $<4000$ \\
\hline 25 & 93162 & WN7+O4f $<49$ & $<360$ & $<280$ & $<10500$ & \\
\hline 26 & MS 1 & WCE/WN5 & $<8.3$ & $<4.3$ & $<28$ & $<88$ \\
\hline 27 & LS 4 & WC6+a & $<2.9$ & $<3.9$ & $<560$ & $<5200$ \\
\hline 28 & MS 2 & WN7 & 0.60 & 1.16 & cnfsd. & cnfsd. \\
\hline 29 & MS 3 & WN7 & $<5.5$ & $<6.2$ & $<45$ & $<130$ \\
\hline 30 & 94305 & WC6+O6-8 & 0.070 & 0.10 & $0.85:$ & $<5.0$ \\
\hline $30 a$ & MS 4 & $\mathrm{WC4}+\mathrm{O4}$ & $<6.7$ & $<8.6$ & $<68$ & $<95$ \\
\hline 31 & 94546 & $\mathrm{WN}_{4}+\mathrm{O} 8 \mathrm{~V}$ & 0.14 & 0.075 & $<2.1$ & $<16$. \\
\hline $31 a$ & SMSP 4 & WC6 & $<0.7$ & $<0.8$ & $<4.7$ & $<43$ \\
\hline 32 & MS 5 & WC5 & 0.15 & 0.20 & 1.68 & $<410$ \\
\hline 33 & 95435 & WC5 & 0.10 & 0.060 & $<5.7$ & $<14$ \\
\hline 34 & LS 5 & WN4.5 & 0.088 & 0.19 & $<430$ & $<1400$ \\
\hline 35 & MS 6 & WN6 & $<21$ & $<81$. & $<320$ & $<750$ \\
\hline $35 a$ & SMSP 5 & WN6 & $<0.06$ & 0.12 & $<60$ & $<150$ \\
\hline $35 b$ & SMSP 6 & WN4 & 0.030 & $<9.3$ & $<190$. & $<240$ \\
\hline 36 & LS 6 & WN4 & 0.058 & 0.15 & $<70$ & $<115$ \\
\hline 37 & MS 7 & WN3 & $<7.5$ & $<27$ & $<240$ & $<250$ \\
\hline 38 & MS 8 & WC4 & $<11$ & $<25$ & $<32$ & $<380$ \\
\hline $\mathbf{3 8 a}$ & SMSP 7 & WN6 & $<14$ & $<32$ & $<290$. & $<370$ \\
\hline $38 \mathbf{b}$ & SMSP 8 & WC7 & 0.13 & $<28$ & $<200$ & $<270$ \\
\hline 39 & MS 9 & WC6 & $<5.9$ & $<20$ & $<120$ & $<170$ \\
\hline 40 & 96548 & WN8(SB1) & 0.69 & 0.28 & $<0.48$ & $<0.12$ \\
\hline 41 & LS 7 & WC6 & 0.27 & $<4.3$ & $<59$ & $<130$ \\
\hline 42 & 97152 & $\mathrm{WC7}+\mathrm{O} 7 \mathrm{~V}$ & 0.28 & 0.073 & $\ldots \ldots$ & $\ldots \ldots$ \\
\hline $42 a$ & SMSP 9 & WN4.5 & 0.10 & $<28$ & $<320$. & $<640$ \\
\hline $42 b$ & SMSP 10 & WN3:+C & 0.13 & $<2.2$ & $<56$ & $<130$ \\
\hline $42 c$ & SMSP 11 & WN6 & $<92$. & $<270$ & $<3600$ & $<5100$ \\
\hline $42 d$ & SMSP 12 & WN4 & $<6000$ & $<39000$. & $<61000$ & $<48000$. \\
\hline 43 & 97950 & WN6+O5 & $<1100$ & $<4300$ & $<5600$ & $<19000$. \\
\hline 44 & LSS 2289 & WN4 & 0.023 & $<0.4$ & $<2.5$ & $<22$ \\
\hline $44 a$ & SMSP 13 & WN3: & 0.86 & 0.38 & $<300$ & $<530$ \\
\hline 45 & LSS 2423 & WC6 & 0.31 & 0.096 & $<1100$ & $<1400$ \\
\hline 46 & 104994 & WN3p & $<34$ & $<14$ & $<44$ & $<85$ \\
\hline 47 & E311884 & WN6+O5V & 0.19 & 0.12 & $<24$ & $<62$ \\
\hline $47 a$ & We 21 & WN8 & 0.29 & 2.15 & 12.96 & $<69$ \\
\hline 48 & 113904 & WC6+09.5I & 0.46 & 0.33 & $<0.9$ & $<5.9$ \\
\hline $48 a$ & Danks 1 & WC8 & $<95$ & $<600$. & $<1600$ & $<1700$. \\
\hline 49 & LSS 2979 & WN5 & 0.20 & 0.20 & $<7.5$ & $<19$ \\
\hline 50 & LSS 3013 & WC6+a & 0.058 & 1.13 & 5.96 & $<88$ \\
\hline & & & 424 & & & \\
\hline
\end{tabular}


TABLE 2-Continued

\begin{tabular}{|c|c|c|c|c|c|c|}
\hline$\overline{\text { WR }}$ & Name(HD) & Spectrum & $\begin{array}{l}\mathrm{S12} \\
\text { [Jy] }\end{array}$ & $\begin{array}{l}\text { S25 } \\
{[\mathrm{Jy}]}\end{array}$ & $\begin{array}{l}\mathrm{S} 60 \\
{[\mathrm{Jy}]} \\
\end{array}$ & $\begin{array}{l}\text { S100 } \\
{[\mathrm{Jy}]}\end{array}$ \\
\hline 51 & LSS 3017 & WN4 & 0.24 & 0.14 & $<260$. & $<330$ \\
\hline 52 & 115473 & WC5 & 0.15 & 0.18 & $<0.8$ & $<11$. \\
\hline 53 & 117297 & WC8 & 0.60 & 0.21 & $<9.6$ & $<46$. \\
\hline 54 & LSS 3111 & WN4 & $<2.6$ & $<0.9$ & $<5.5$ & $<12$. \\
\hline 55 & 117688 & WN7 & 0.39 & $<2.4$ & $<11$. & $<71$ \\
\hline 56 & LS 8 & WC7 & $<1.0$ & $<3.5$ & $<3.8$ & $<24$. \\
\hline 57 & 119078 & WC7 & 0.098 & 0.13 & $<0.8$ & $<1.8$ \\
\hline 58 & LSS 3162 & WN4/WCE & $<0.4$ & $<0.5$ & $<3.9$ & $<9.4$ \\
\hline 59 & LSS 3164 & WC9 & 0.60 & $<20$ & $<190$. & $<710$. \\
\hline 60 & 121194 & WC8 & 0.42 & 0.19 & $<25$. & $<150$. \\
\hline 61 & LSS 3208 & WN6 & 0.023 & 0.069 & $<4.7$ & $<27$. \\
\hline 62 & NS 2 & WN6 & 0.30 & 0.089 & $<32$ & $<130$. \\
\hline 63 & LSS 3289 & WN6 & 0.56 & 0.36 & $<380$. & $<860$. \\
\hline 64 & BS 3 & WC7 & 0.17 & 0.049 & $<4.3$ & $<22$ \\
\hline 65 & LSS 3319 & WC9 & $<38$. & $<33$ & $<250$ & $<660$. \\
\hline 66 & 134877 & WN8 & 0.064 & 0.083 & 0.29 & $<140$ \\
\hline 67 & LSS 3329 & WN6 & 0.20 & $<5.7$ & $<49$. & ....... \\
\hline 68 & BS 4 & WC7 & 0.068 & 0.11 & $<70$ & $<160$. \\
\hline 69 & 136488 & WC9 & 0.99 & 0.29 & 0.67 & $<11$. \\
\hline 70 & 137603 & WC9+BoI & 6.53 & 2.03 & 0.75 & $<13$ \\
\hline 71 & 143414 & WN6(SB1) & 0.072 & 0.073 & 0.44 & 3.98: \\
\hline 73 & NS 3 & WC9 & 0.53 & 0.21 & 1.01 & 3.55 \\
\hline 74 & BP 1 & WN7 & $<107$ & $<80$ & $<34$ & $<73$ \\
\hline 75 & 147419 & WN5 & 0.28 & $<24$. & $<180$. & $<220$ \\
\hline 76 & LSS 3693 & WC9 & 16.46 & 26.77 & 66.65: & $<6800$ \\
\hline 77 & He3-1239 & WC8 $(+a)$ & $<2.8$ & $<2.7$ & $<6.0$ & $<150$ \\
\hline 78 & 151932 & WN7 & 1.30 & 0.58 & $<4.9$ & $<43$ \\
\hline 79 & 152270 & $\mathrm{WC} 7+05-8$ & 0.69 & 0.42 & $<14$. & $<42$. \\
\hline 80 & LSS 3871 & WC9 & 1.79 & 0.47 & $<13$ & $<58$ \\
\hline 81 & He3-1316 & WC9 & 0.36 & 0.20 & $<28$. & $<65$ \\
\hline 82 & LS 11 & WN8 & 0.23 & 0.20 & $<12$. & $<59$ \\
\hline 83 & He3-1344 & WN6 & 0.080 & 0.092 & 0.15 & $<53$ \\
\hline 84 & The 3 & WN6 & 0.27 & $<26$ & $<280$. & $<540$ \\
\hline 85 & LSS 3982 & WN6 & $<55$ & $<38$ & $<6.2$ & $<91$. \\
\hline 86 & 156327 & $\mathbf{W C 7}+\mathbf{a}$ & 0.26 & 0.22 & 1.60 & $<31$. \\
\hline 87 & LSS 4064 & WN7 & 0.77 & 3.72 & 14.17 & $<20$ \\
\hline 88 & The 1 & WC9 & 1.45 & $<47$. & $<220$. & $<170$ \\
\hline 89 & LSS 4065 & WN7 & 0.39 & 3.36 & 21.59 & $<260$ \\
\hline 90 & 156385 & WC7 & 0.90 & 0.40 & 0.30 & $<6.2$ \\
\hline 91 & StSa 1 & WN7 & $<2500$ & $<14000$. & $<44000$ & $<34000$ \\
\hline 92 & 157451 & WC9 & 0.06 & 0.18 & $<1.0$ & $<5.1$ \\
\hline 93 & 157504 & WC $7+07-9$ & $<8200$ & cnfsd. & cnfsd. & cnfsd. \\
\hline $93 a$ & PKS359+3.1 & WN2.5-3 & 0.055 & 0.10 & $<1.0$ & $<9.9$ \\
\hline 94 & 158860 & WN6 & 0.31 & $<6.5$ & $<100$. & $<190$ \\
\hline 95 & He3-1434 & WC9 & 4.34 & 3.39 & 2.92 & $<200$ \\
\hline 96 & LSS 4265 & WC9 & 1.68 & 0.66 & $<32$. & $<150$ \\
\hline 97 & E320102 & WN3+O5-7 & 0.089 & 0.21 & 1.38 & $<30$. \\
\hline 98 & E318016 & WC7/WN6 & 0.54 & $<1.6$ & $<44$. & $<120$ \\
\hline $98 \mathbf{a}$ & IRAS 17380 & WC9 & 50.34 & 13.80 & 18.91 & $<410$ \\
\hline 100 & E318139 & WN6 & 0.42 & 0.22 & 0.39 & $<17$ \\
\hline 101 & DA 3 & WC8 & $<5.0$ & $<7.0$ & 37.61 & $<110$ \\
\hline 102 & Sand 4 & W01 & $<3.0$ & 8.92 & 32.56 & $<220$ \\
\hline 103 & 164270 & WC9 (SB1?) & 0.97 & 0.33 & $<0.9$ & $<9.0$ \\
\hline 104 & Ve2-45 & WC9 & 406.19 & 135.25 & 19.94 & $<330$ \\
\hline 105 & NS 4, Ve2-47 & WN8 & 1.80 & 1.32 & $<7.1$ & $<170$ \\
\hline 106 & E313643 & WC9 & 11.51 & 3.88 & 14.68 & $<100$ \\
\hline 107 & DA 1 & WN8 & $<6.2$ & $<2.7$ & cnfsd. & cnfsd. \\
\hline 108 & E313846 & WN9 & 0.37 & 0.14 & $<48$ & $<210$ \\
\hline 109 & NS 5 & WN3 & $<0.2$ & $<0.3$ & $<0.4$ & $<6.6$ \\
\hline 110 & 165688 & WN6 & 0.39 & $<15$. & $<200$ & $<650$ \\
\hline 111 & 165763 & WC5 & 0.79 & 0.47 & $<17$. & $<37$ \\
\hline 112 & GL 2104 & WC9 & 153.59 & 73.35 & 19.27 & 7.33 \\
\hline 113 & 168206 & WC8+08-9IV & 2.35 & 2.13 & $<120$. & $<630$. \\
\hline 114 & 169010 & WC5 & $<34$ & $<25$. & $<63$. & $<120$ \\
\hline 115 & IC14-19 & WN6 & 4.73 & 3.96 & 2.81 & $<16$. \\
\hline 116 & ST 1 & WN8 & 0.80 & 7.81 & $<37$ & $<190$ \\
\hline
\end{tabular}


TABLE 2-Continued

\begin{tabular}{|c|c|c|c|c|c|c|}
\hline$\overline{\text { WR }}$ & Name(HD) & Spectrum & $\begin{array}{l}\mathrm{S12} \\
{[\mathrm{Jy}]}\end{array}$ & $\begin{array}{l}\text { S25 } \\
\text { [Jy] }\end{array}$ & $\begin{array}{l}\mathrm{S} 60 \\
\text { [Jy] }\end{array}$ & $\begin{array}{c}\text { S100 } \\
{[\mathrm{Jy}]}\end{array}$ \\
\hline 117 & IC14-22 & WC8 & 0.28 & 0.13 & $<5.1$ & $<16$. \\
\hline 118 & GL 2179 & WC9 & 70.32 & 21.67 & 34.59 & 105.25 \\
\hline 119 & The 2 & WC9 & 0.62 & 0.22 & $<32$ & $<115$. \\
\hline 120 & Vy1-3 & WN7 & 0.27 & $<11$ & $<110$ & $<640$. \\
\hline 121 & AS 320 & WC9 & 2.61 & 1.34 & $1.77:$ & $<180$. \\
\hline 123 & 177230 & WN8 (SB1) & 0.090 & 0.071 & $<1.5$ & $<3.0$ \\
\hline 125 & IC14-36 & WC7 & 0.95 & 1.48 & $<210$ & $<960$. \\
\hline 126 & ST 2 & WC5/WN & 0.065 & 0.035 & $<1.4$ & $<7.0$ \\
\hline 127 & 186943 & WN4+09.5V & 0.041 & $<3.6$ & $<26$ & $<27$. \\
\hline 128 & 187282 & WN4 (SB1) & 0.037 & 0.045 & 0.13 & $<3.0$ \\
\hline 130 & LS 16 & WN8 & 1.55 & 12.50 & 41.15 & 33.29 \\
\hline 131 & IC14-52 & WN7+a & $<0.6$ & $<2.2$ & $<36$ & $<100$. \\
\hline 132 & 190002 & WC6 & $<0.6$ & $<0.2$ & $<3.7$ & $<17$ \\
\hline 133 & 190918 & WN4.5+09.5Ib & 0.34 & 0.12 & 0.42 & $<6.4$ \\
\hline 134 & 191765 & WN6 (SB1) & 0.65 & 0.37 & $0.35:$ & $<1.0$ \\
\hline 135 & 192103 & WC8 & 0.22 & 0.17 & 0.31 & $<55$ \\
\hline 136 & 192163 & WN6 (SB1) & 1.33 & 0.55 & $<6.4$ & $<51$. \\
\hline 137 & 192641 & WC7+OB & 3.14 & 2.42 & 20.00 & 64.73 \\
\hline 138 & 193077 & WN6+O9 & 0.41 & 0.23 & 0.12 & $<5.2$ \\
\hline 139 & 193576 & WN5+O6 & 0.59 & 0.30 & 0.11 & $<39$. \\
\hline 140 & 193793 & $W C 7+04-5$ & 1.82 & 1.00 & $<1.2$ & $<46$. \\
\hline 141 & 193928 & WN6(SB1) & $<840$ & $<240$. & $<51$ & $<93$. \\
\hline 142 & ST 3 & WO2 & $<22$ & $<24$ & $<51$ & $<730$. \\
\hline 143 & 195177 & WC5 & 0.29 & 0.32 & 3.38 & $<43$. \\
\hline 144 & HM19-1 & WC4 & 0.37 & 0.38 & $<260$. & $<320$. \\
\hline 145 & AS 422 & WN3/WCE(SB1) & 0.59 & 0.22 & $0.23:$ & $<330$. \\
\hline 146 & HM19-3 & WC6 & 0.95 & $<2.2$ & $<20$ & $<190$. \\
\hline 147 & NS 6 & WN8 & 5.18 & 3.08 & 1.35 & $<110$. \\
\hline 148 & 197406 & WN7 (SB1) & 0.11 & $0.05:$ & $<1.8$ & $<4.2$ \\
\hline 149 & ST 4 & WN6-7 & 0.080 & 0.067 & $<2.0$ & $<10$. \\
\hline 150 & ST 5 & WC5 & 0.075 & 0.060 & 0.30 & 3.40 \\
\hline 151 & CX Cep & WN4+O8V & 0.095 & 0.033 & $<2.7$ & $<9.6$ \\
\hline 152 & 211564 & WN3 & $<2.8$ & $<3.5$ & $<27$ & $<58$. \\
\hline 153 & 211853 & WN6/WCE+O & 0.25 & $<75$ & $<440$. & $<600$. \\
\hline 154 & 213049 & WC6 & 0.10 & 0.036 & $<2.1$ & $<6.8$ \\
\hline 155 & 214419 & WN7+O & 0.16 & 0.083 & $<0.9$ & $<6.9$ \\
\hline 156 & $\mathrm{AC}+6038562$ & WN8 & 0.33 & 0.26 & 0.41 & 0.33 \\
\hline 157 & 219460 & WN4.5 $(+B)$ & 0.17 & 0.094 & $<49$ & $<75$. \\
\hline 158 & AS 513 & WN7 & 0.18 & 0.11 & 0.13 & cnfsd. \\
\hline
\end{tabular}

TABLE 3

IRAS COLORS OF NONDUSTY Single and BINARY WOL.F-RAYET STARS

\begin{tabular}{llcc}
\hline \hline Sample & WR type & {$[12]-[25]$} & {$[25]-[60]$} \\
\hline Morris et al. & WN - single & $1.32 \pm 0.18(16)$ & $3.00 \pm 0.35(9)$ \\
Morris et al. & WN - binary & $1.10 \pm 0.16(9)$ & $1.98 \pm 0.46(5)$ \\
Morris et al. & WC - single & $1.26 \pm 0.19(12)$ & $2.33 \pm 0.51(4)$ \\
Morris et al. & WC - binary & $1.76 \pm 0.57(7)$ & $3.71 \pm 0.28(5)$ \\
& & & \\
Morris et al. & all WRs - single & $1.30 \pm 0.13(28)$ & $2.80 \pm 0.29(13)$ \\
Morris et al. & all WRs - binary & $1.39 \pm 0.27(16)$ & $2.84 \pm 0.38(10)$ \\
& & & \\
HHASD & WN - single & $1.88 \pm 0.18(33)$ & $2.92 \pm 0.28(15)$ \\
HHASD & WN - binary & $1.11 \pm 0.12(21)$ & $2.56 \pm 0.31(11)$ \\
HHASD & WC - single & $1.22 \pm 0.14(22)$ & $3.40 \pm 0.44(6)$ \\
HHASD & WC - binary & $1.52 \pm 0.41(10)$ & $3.29 \pm 0.48(6)$ \\
& & & \\
HHASD & all WRs - single & $1.62 \pm 0.13(55)$ & $3.04 \pm 0.24(21)$ \\
HHASD & all WRs - binary & $1.24 \pm 0.16(31)$ & $2.82 \pm 0.27(17)$ \\
\hline
\end{tabular}


TABLE 4

IR AS COLORS OF DIFFERENT TYPES OF WOLF-RAYET STARS

\begin{tabular}{|c|c|c|c|}
\hline WR type & {$[12]-[25]$} & {$[25]-[60]$} & {$[60]-[100]$} \\
\hline WN2 & $2.30(1)$ & $2.81(1)$ & $(0)$ \\
\hline WN2.5 & $2.26(1)$ & $(0)$ & (0) \\
\hline WN3 & $1.72 \pm 0.54(4)$ & $2.89 \pm 0.59(3)$ & (0) \\
\hline WN4 & $1.67 \pm 0.43(6)$ & $4.14 \pm 1.07(2)$ & $1.94(1)$ \\
\hline WN4.5 & $1.45 \pm 0.34(5)$ & $2.99 \pm 0.58(3)$ & $2.25(1)$ \\
\hline WN5 & $1.12 \pm 0.13(5)$ & $1.52 \pm 0.72(2)$ & (0) \\
\hline WN6 & $1.24 \pm 0.18(12)$ & $2.28 \pm 0.39(6)$ & $3.71(1)$ \\
\hline WN7 & $1.78 \pm 0.44(8)$ & $3.16 \pm 0.56(3)$ & $(0)$ \\
\hline WN8 & $1.96 \pm 0.39(11)$ & $2.80 \pm 0.49(5)$ & $1.08 \pm 0.01(2)$ \\
\hline WN9 & $0.54(1)$ & $(0)$ & (0) \\
\hline All WNs & $1.56 \pm 0.12(54)$ & $2.76 \pm 0.21(25)$ & $2.01 \pm 0.48(5)$ \\
\hline WO1 & (0) & $3.36(1)$ & (0) \\
\hline WO2 & (0) & $(0)$ & (0) \\
\hline WC4 & $1.85 \pm 0.13(3)$ & $3.75(1)$ & $(0)$ \\
\hline WC5 & $1.33 \pm 0.13(10)$ & $3.31 \pm 0.57(5)$ & $3.95(1)$ \\
\hline WC6 & $1.46 \pm 0.18(8)$ & $3.88 \pm 0.18(3)$ & (0) \\
\hline WC7 & $1.19 \pm 0.22(10)$ & $3.34 \pm 0.85(3)$ & $2.59(1)$ \\
\hline WC8 & $0.91 \pm 0.17(6)$ & $1.90 \pm 0.70(2)$ & $0.96(1)$ \\
\hline WC9 & $0.76 \pm 0.17(17)$ & $2.08 \pm 0.36(11)$ & $1.82 \pm 0.78(3)$ \\
\hline All WCs & $1.12 \pm 0.11(54)$ & $2.79 \pm 0.26(25)$ & $2.16 \pm 0.54(6)$ \\
\hline All WRs & $1.34 \pm 0.09(108)$ & $2.78 \pm 0.16(51)$ & $2.09 \pm 0.35(11)$ \\
\hline All free-free WRs & $1.46 \pm 0.10(87)$ & $2.58 \pm 0.28(39)$ & $2.14 \pm 0.47(7)$ \\
\hline Non-dusty WCs & $1.31 \pm 0.15(33)$ & $3.19 \pm 0.32(13)$ & $2.45 \pm 1.49(2)$ \\
\hline All DWCLs & $0.84 \pm 0.13(21)$ & $2.35 \pm 0.39(12)$ & $2.01 \pm 0.58(4)$ \\
\hline
\end{tabular}

same analysis of IRAS colors, but now based on HHASD's designations of single and binary for the entire sample of W-R stars in Table 2. I dropped the hybrid stars from consideration in this context because of their potentially ambiguous interpretation as single or composite stars, but assigned to the binaries any W-R star with a type of "SB1" or "SB2" ( spectroscopic binaries ), or "+O" (companion assigned a spectral type, usually O type), or "+a" (absorption lines detected in the spectrum). Although single WN stars tend to be redder in the mean than binaries, neither WC stars alone nor the sample of all W-R stars can sustain any statistically significant distinction between the (mean $\pm 3 \sigma$ ) color ranges of single and binary systems.

Consequently, Table 4 summarizes the separate average [12] - [25] colors for W-R stars of all subtypes, distinguishing WNs from WCs. The colors [25]-[60] and [60]-[100] are essentially all the same for WN and WC types and show no trends. Hybrid stars were assigned to the category corresponding to their dominant subtype (that given first). The average for all WNs is [12] $-[25]=1.56 \pm 0.12$ ( 54 stars), [25] $[60]=2.76 \pm 0.21$ (25 stars $)$; for all WCs it is $1.12 \pm 0.11(54$ stars) and $2.79 \pm 0.26$ ( 25 stars), respectively.

These colors indicate a definite "blueward" trend with lateness of subtype for [12]- [25] among the WC stars, whereas WNs show no pattern (Fig. 4). To sharpen the search for DWCLs, I also tabulate the colors for known DWCLs based on the set of 25 WCs established to show either permanent or variable dust emission (see Williams \& van der Hucht 1992). I have IRAS data for 21 of these stars that define a DWCL color zone centered on $[12]-[25]=0.84 \pm 0.13,[25]-[60]=$
$2.35 \pm 0.39,[60]-[100]=2.01 \pm 0.58$. The average for all $\mathrm{WN}, \mathrm{WO}$, and WC stars is [12] $-[25]=1.34 \pm 0.09(108$ stars) and $[25]-[60]=2.78 \pm 0.16$ (51 stars)

By removing all these DWCLs, one can define the colors of nondusty WCLs too (Table 4). The WNs, early-type WCs (WCEs), and nondusty WCLs show no statistically significant color differences, suggesting that all these stars are dominated by a common emission mechanism at least from 12 to $100 \mu \mathrm{m}$.
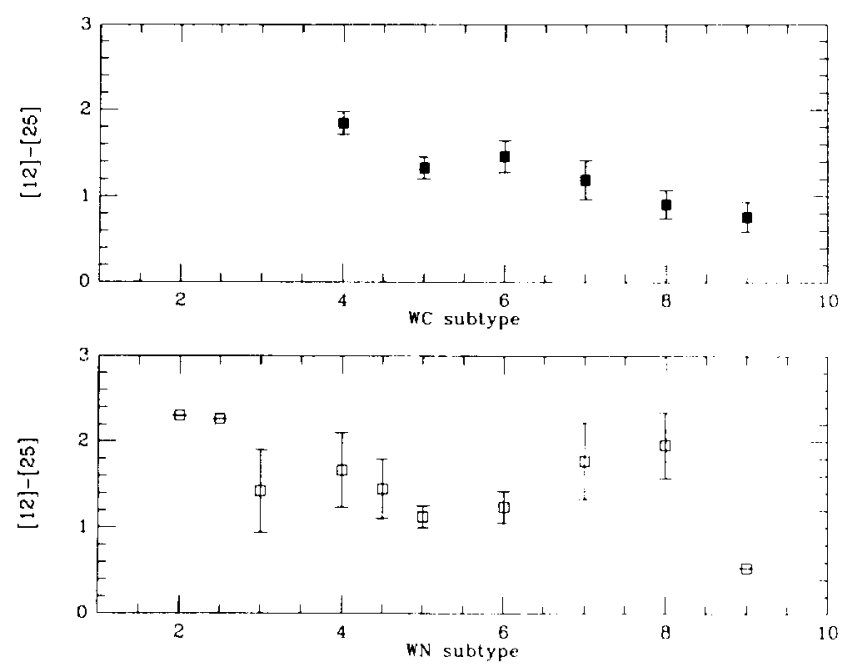

FIG. 4.-Mean IRAS [12]- [25] colors of W-R stars, distinguishing WC and WN types (see Table 4). 
Consequently, I have also created colors for the ensemble of "all others," meaning all W-R stars excluding only DWCLs (the two WO stars are included here too). Thus there are two separate color-color zones to represent DWCLs and all other W-R stars. The hatched rectangle in Figure 3 corresponds to the 12-25-60 $\mu \mathrm{m}$ zone characteristic for DWCLs (with extent in each axis of $\pm 1.39 \sigma$ about the mean color). The crosshatched rectangle in Figure 3 likewise represents all nondusty W-R stars. (Note that Fig. 3 is not identical to the Walker et al. 1989 diagram. They used non-color-corrected colors, whereas I have presented Fig. 3 in the same context as the W-R colors, namely, with hot color correction: the differences in occupation zone boundaries amount to only $0.03 \mathrm{mag}$ in [12] - [25] and $0.07 \mathrm{mag}$ in [25] - [60].)

Morris et al. ( 1993) found the mean spectral index characterizing the short-wavelength power laws of W-R stars to be -2.85 [in $F_{\lambda}(\lambda)$ terms], corresponding to a color index, [12] - [25], of 0.95. None of the mean color indices in Table 3 is as small as this value, although there are certainly some individual stars with colors near 0.95 and even lower values too. Morris et al. demonstrated that their power-law spectra appear to continue to $25 \mu \mathrm{m}$ for the three WN stars studied using IRAS data by Mathis et al. (1992), namely, W-R 6, $\mathrm{W}-\mathrm{R} 40$, and $\mathrm{W}-\mathrm{R} 136$. These $I R A S$ spectral indices were estimated as $-2.7,-3.2$, and -3.0 . My own values of [12] $-[25]$ imply spectral indices for these stars of $-2.7,-3.2,-3.2$, in close agreement with Mathis et al. However, most W-R stars show slopes closer to -2.0 between 12 and $25 \mu \mathrm{m}$, such as optically thin free-free emission from their stellar winds would produce. They are close to a slope of -2.8 between 25 and 60 $\mu \mathrm{m}$ on the basis of their mean color indices, probably indicative of increasing optical depth at longer wavelengths. Morris et al. (1993) concluded that there must be a substantial contribution from free-free emission to the short-wavelength continua of W-R stars. It would take a detailed side-by-side investigation of W-R stars to establish whether exactly the same power-law continuum found for $W$ - $R$ stars between the ultraviolet and the near-infrared extends into the $I R A S$ wavelength region. For the present we note simply that all nondusty $W-R$ stars appear to be consistent with a continuum, largely due to free-free emission, that stretches from the ultraviolet into the IRAS wavelength range.

One can understand the differences in color and even intuit approximately where the W-R populations should lie. Monochromatic flux densities for zero magnitude, $\mathrm{ZMF}_{\lambda}$, are defined by the photospheric spectrum of a hot star such as Sirius, falling roughly as $\lambda^{-4}$. Thus, the ratio $\left(\mathrm{ZMF}_{12} / \mathrm{ZMF}_{25}\right) \sim$ 18.8. Assuming ( 1 ) that all nondusty single $W-R$ stars are characterized by simple power laws, as Morris et al. have established, at least for single stars, (2) that the IRAS measurements of binary W-R stars are negligibly contaminated by photospheric radiation from any secondary, and (3) that the typical slope of these power laws is dictated by optically thin free-free emission which has $F_{\lambda} \propto \lambda^{-2}$, one would expect a free-free flux density ratio, $F_{12} / F_{25}$, of $\sim 4.3$. Therefore, the free-free color index is expected to be

$[12]-[25]$

$$
=-2.5 \log \left[\left(F_{12} / F_{25}\right) /\left(\mathrm{ZMF}_{25} / \mathrm{ZMF}_{12}\right)\right], \quad \text { or } \sim 1.6 .
$$

Similarly, W-R stars dominated by thermal emission from hot dust have not attained their Rayleigh-Jeans domain, so $F_{\lambda} \propto$ $\lambda^{-3}$, hence the flux density ratio $F_{12} / F_{25}$ should be $\sim 9.0$, leading to a dust color index of $[12]-[25] \sim 0.8$. From Tables 3 and 4 one finds that the free-free dominated W-R stars have about 1.5 as their mean value of [12]- [25], while the DWCLs (Table 4) have [12] - [25] of about 0.8 , both similar to these rough estimates, reinforcing this interpretation of the IRAS energy distributions of nondusty and dusty W-R stars. (The actual mean value of [12] - [25] observed for all nondusty W-R stars, namely, 1.46, corresponds to a power law with slope -2.2 , still quite close to optically thin free-free.)

\section{THE SEARCH FOR NEW WOLF-RAYET CANDIDATES}

\subsection{DWCLS}

Note (Fig. 3) that DWCLs are essentially distinct from all other types of source, while the main body of W-R stars-those emitting predominantly free-free radiation; see below-overlaps considerably the zone for "blue planetary nebulae," and to a lesser extent that for "T Tauri stars" ( see also Walker et al. 1989 , their Table 1). This relative isolation suggests the potential value of a color-color search of the PSC. The specific "core query" made to identify new DWCL candidates was $0.649 \leq$ $[12]-[25] \leq 1.024,1.809 \leq[25]-[60] \leq 2.885,|b|<5^{\circ}$, with no associations to catalogs other than $I R A S$, and having FQUAL $=333^{*}$. Very few W-R stars are detected at $100 \mu \mathrm{m}$, and there is always danger of cirrus contamination, so I chose not to use the additional $[60]-[100]$ color constraint, to avoid possible rejection of otherwise viable W-R candidates. (In fact, virtually all those candidates that passed the LRS shape criterion were undetected at $100 \mu \mathrm{m}$ and would, indeed, have been lost at this stage, had the third color been required.)

The definition of an occupation zone implies that only $\sim 70 \%$ of any normally distributed population lies in the 2.78 $\sigma$ wide box. I would like to be more complete for the DWCLs; therefore, I doubled the color zone searched to $0.461 \leq[12]-$ $[25] \leq 1.211,1.271 \leq[25]-[60] \leq 3.423$, corresponding to over $99 \%$ of a normally distributed population. There is, of course, no guarantee that the rather limited number of W-R stars in the Galaxy are normal in their color distributions, so this more conservative approach seems justified. I also included as candidates any PSC source whose mean IRAS colors lay outside this broader search area but whose colors overlapped the query box at the mean $\pm 1 \sigma$ level, using the actual uncertainties in the source colors, as constructed from the relevant pairs of PSC "RELUNCs."

The broader INGRES query on the PSC using these criteria yielded 219 candidates. I extracted all LRS spectra for each of these candidates, finding spectra to exist for 132 sources (a total of over 500 individual spectra, typically four per candidate). Many objects are, of course, very faint to IRAS, and their LRS spectra are correspondingly noisy. However, a total of 13 IRAS sources (in both hemispheres) was returned, having LRS spectral signatures quantitatively like those of the known DWCLs. Their locations in the sky need to be examined for optical counterparts, then pursued spectroscopically (or at least using pairs of narrow passbands isolating WC line and continuum regions in a $C C D$ survey). $A$ viable alternative may be near-infrared low-resolution spectroscopy ( near $2 \mu \mathrm{m}$ ) 

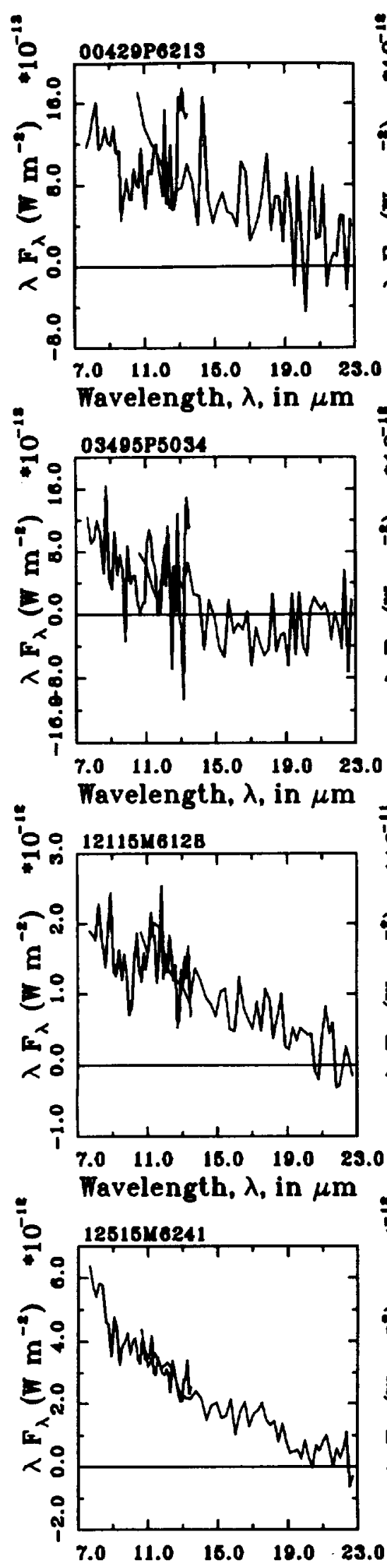

Wavelength, $\lambda$, in $\mu \mathrm{m}$
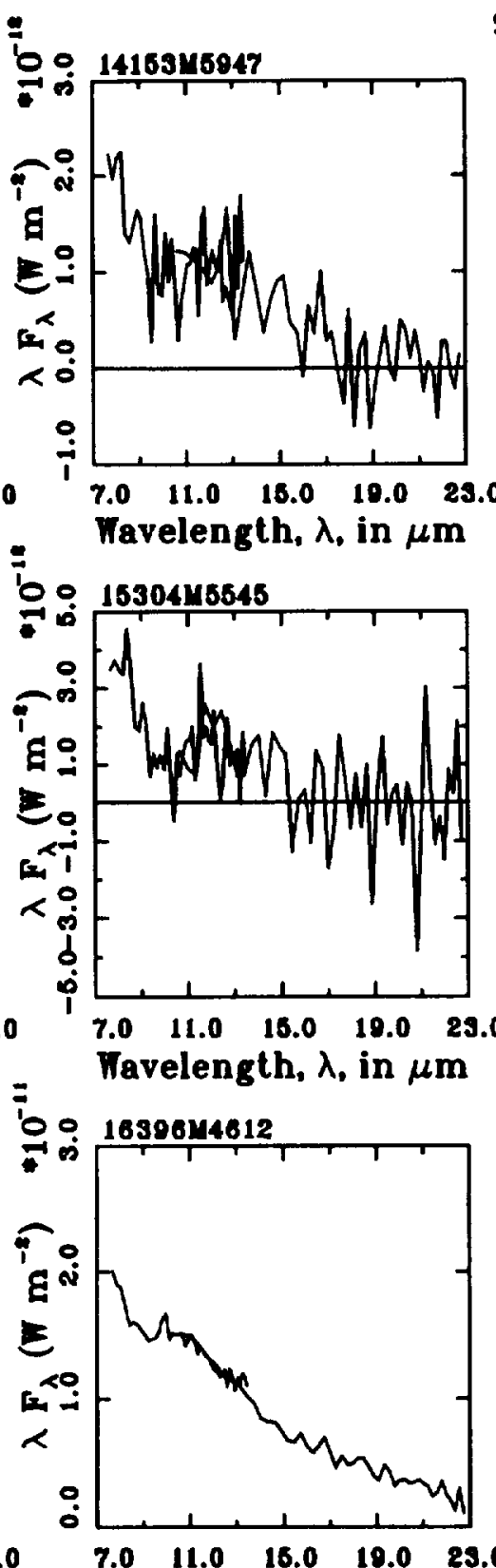

$\begin{array}{lllll}7.0 & 11.0 & 16.0 & 19.0 & 29.0\end{array}$
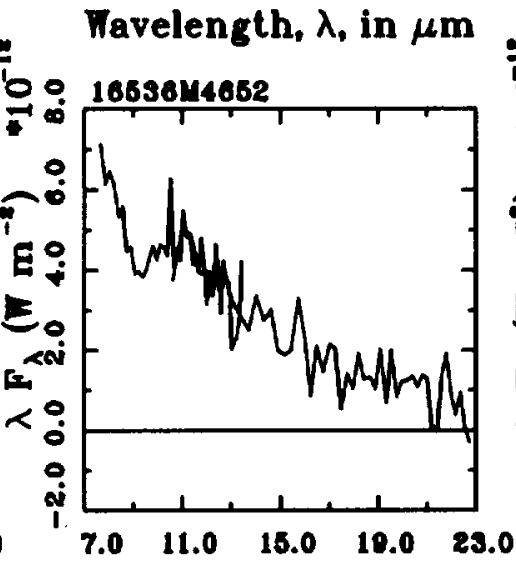

Favelength, $\lambda$, in $\mu \mathrm{m}$

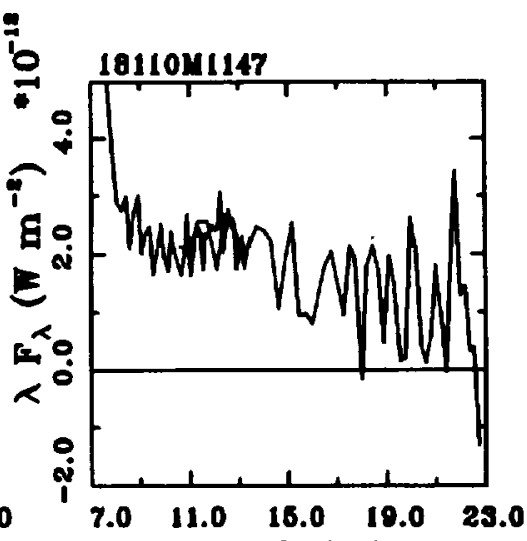

Wavelength, $\lambda_{\text {, in } \mu \mathrm{m}}$

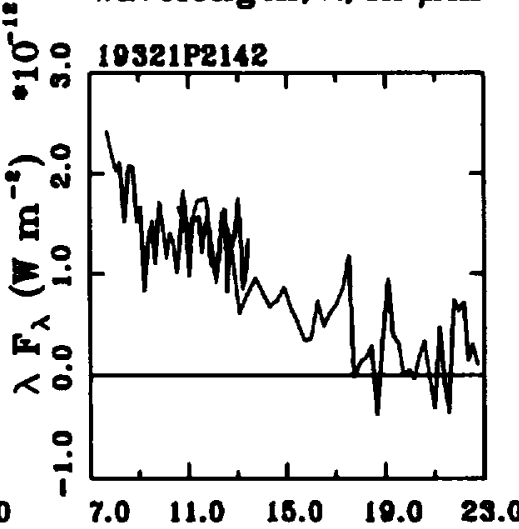

Navelength, $\lambda_{1}$ in $\mu \mathrm{m}$

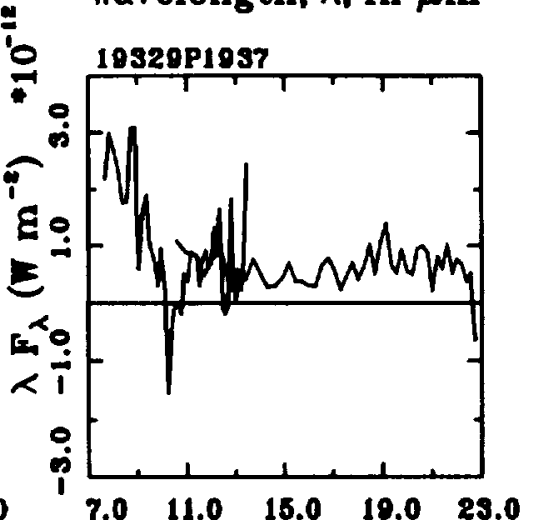

Favelength, $\lambda$, in $\mu \mathrm{m}$

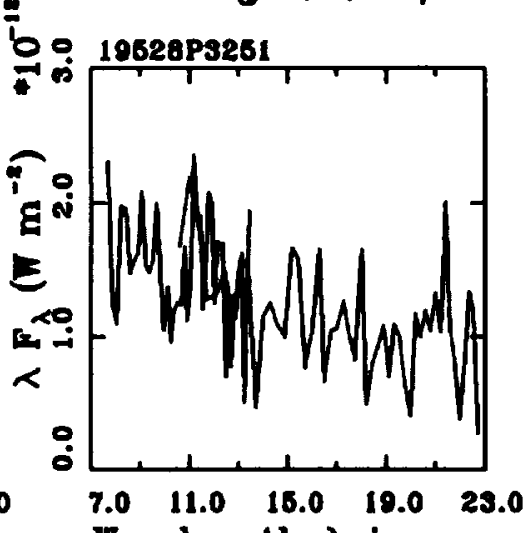

Tavelength, $\lambda$, in $\mu \mathrm{m}$

FIG. 5.-LRS spectra of the 13 DWCL candidates 


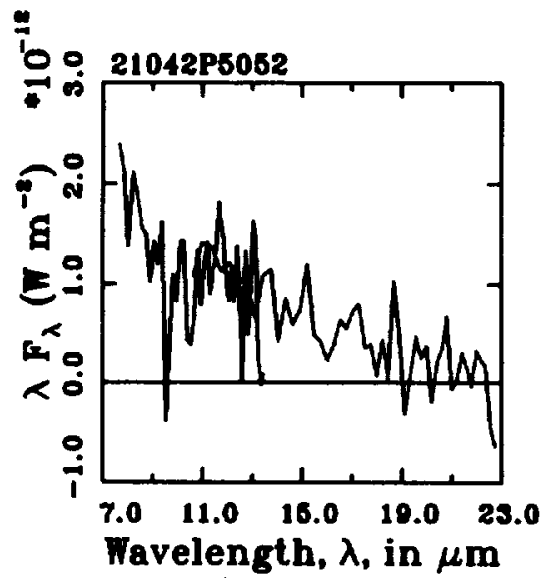

FIG. 5-Continued

that is readily capable of detecting the broad bright emission lines of W-R stars (cf. Eenens et al. 1991). Table 5 gives the $I R A S$ names and 1950 coordinates of these 13 sources, four of which (those with asterisks in the table) were returned by the core query $[ \pm 1.39 \sigma]$ and the remaining nine by the broader search $[ \pm 2.78 \sigma])$. Figure 5 plots their $I R A S$ LRS spectra (compare with Fig. 2).

\subsection{Other Wolf-Ravet Stars}

Identically constructed queries on the PSC, with core and doubly broadened searches using the occupation zone for all other ( free-free-emitting) W-R stars (Table 4), resulted in 70 sources being returned, of which 28 have an LRS spectrum. Only one of these, however, looked quantitatively like a freefree spectrum, namely, that for IRAS $19537+2608$. Figure 6 presents this LRS spectrum, and its coordinates are appended to Table 5. This single source emerged from the core query, $1.318 \leq[12]-[25] \leq 1.588,2.182 \leq[25]-[60] \leq 2.918$;

TABLE 5

NAMES AND COORDINATES OF IRAS DWCL AND FREE-FREE EMISSION CANDIDATES

\begin{tabular}{lcl}
\hline \hline IRASNAME & RA(1950) & DEC (1950) \\
\hline DWCLs & & \\
$00428+6213$ & $00^{h} 42^{m} 53.6^{*}$ & $+62^{\circ} 13^{\prime} 33^{\prime \prime}$ \\
$03495+5034$ & 034931.6 & +503416 \\
$12114-6128^{*}$ & 121130.0 & -612831 \\
$12515-6241$ & 125132.8 & -624154 \\
$14153-5947^{*}$ & 141522.7 & -594732 \\
$15304-5545^{*}$ & 153024.4 & -554518 \\
$16396-4613$ & 163939.4 & -461307 \\
$16538-4652$ & 165351.4 & -465240 \\
$18110-1148$ & 181101.9 & -114813 \\
$19321+2141$ & 193211.1 & +214154 \\
$19329+1937^{*}$ & 193258.5 & +193725 \\
$19528+3251$ & 195249.5 & +325138 \\
$21041+5052$ & 210411.5 & +505232 \\
Free-free WRs & & \\
$19537+2608^{*}$ & 195343.9 & +260841 \\
\hline
\end{tabular}

a IRASNAMEs with asterisks indicate sources returned by the "core" queries; others come from the broader searches. no other additional free-free-dominated $\mathbf{W}$ - $R$ candidates were found in the broader search.

\section{NATURE OF THE ENERGY DISTRIBUTIONS OF WOLF-RAYET STARS}

It is instructive to examine the mean colors for DWCLs and all nondusty W-R stars in terms of their implied $I R A S$ energy distributions. To do this, one normalizes each energy distribution at $12 \mu \mathrm{m}$ and calculates the successive $F_{\lambda}$ values via the magnitudes at 25,60 , and $100 \mu \mathrm{m}$ derived from the color indices. The DWCL spectrum can be broken into a $500 \mathrm{~K}$ blackbody that fits the entire flux at 12 and $25 \mu \mathrm{m}$, and about $17 \%$ of that at $60 \mu \mathrm{m}$, and a $38 \mathrm{~K}$ blackbody to account for the remaining $60 \mu \mathrm{m}$ and all the $100 \mu \mathrm{m}$ fluxes. The rough characteristic scale of the $500 \mathrm{~K}$ dust envelope is about $3600 R_{\text {* }}$ from the WC star's surface. Assuming that the long-wavelength component is not simply cirrus contamination then, if the cold dust were also heated by direct but diluted starlight, it would lie at about $2.8 \times 10^{5} R_{*}$. This component could represent either interstellar material swept up by the W-R stellar wind or conceivably the remnant of an ancient episode of dust formation.

Analysis of a similarly constructed average energy distribution for all nondusty W-R stars indicates that, if one attributes the 12 and $25 \mu \mathrm{m}$ fluxes entirely to optically thin free-free emission, there is still significant long-wavelength radiation that is not accounted for (if one uses a steeper power law such as Morris et al. 1993 find typical of W-R stars at shorter wavelengths, there is already an excess at $25 \mu \mathrm{m}$, hence my use of the flatter optically thin spectrum). This has a color temperature of $\sim 45$ $\mathrm{K}$ and might again be dusty interstellar material swept up by the powerful stellar winds, whether or not it is associated with a visible nebula or "bubble" (as are many W-R stars).

\section{COMPLETENESS OF THE SEARCH FOR NEW WOLF-RAYET STARS}

By using distances from Conti \& Vacca (1990) (who used a homogeneous set of intrinsic colors to determine W-R dis-

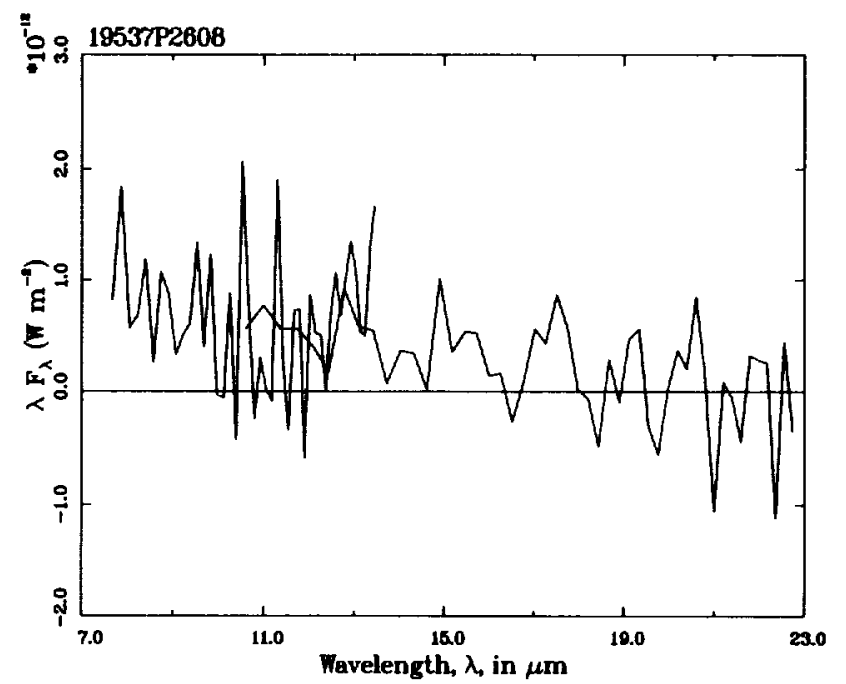

FIG. 6.-LRS spectrum of the single free-free-dominated W-R candidate. 
TABLE 6

ARSOLUTE IRAS MAGNITUDES OF NONDUSTY SINGLE AND BINARY WOLF-RAYET STARS

\begin{tabular}{lccc}
\hline WR sample & $\mathrm{M}_{12}$ & $\mathrm{M}_{25}$ & $\mathrm{M}_{60}$ \\
\hline WN - single & $-7.21 \pm 0.17(39)$ & $-9.08 \pm 0.26(32)$ & $-12.18 \pm 0.45(14)$ \\
WN - binary & $-6.75 \pm 0.22(23)$ & $-7.74 \pm 0.26(20)$ & $-10.15 \pm 0.63(10)$ \\
WC - single & $-6.96 \pm 0.26(23)$ & $-7.93 \pm 0.21(19)$ & $-10.71 \pm 0.53(5)$ \\
WC - binary & $-6.01 \pm 0.22(11)$ & $-7.55 \pm 0.50(10)$ & $-11.06 \pm 0.94(7)$ \\
& & & \\
All WRs - single & $-7.12 \pm 0.14(62)$ & $-8.65 \pm 0.20(51)$ & $-11.80 \pm 0.38(19)$ \\
All WRs - binary & $-6.51 \pm 0.17(34)$ & $-7.68 \pm 0.24(30)$ & $-10.52 \pm 0.53(17)$ \\
\hline
\end{tabular}

tances via reddening), in conjunction with Table 2 , absolute IRAS magnitudes of $\mathrm{W}-\mathrm{R}$ stars were also defined, to assess the volume likely to have been sampled by $I R A S$. I excluded Conti \& Vacca's ( 1990) distances of $34 \mathrm{kpc}$ for WR $64=\mathrm{BS} \mathrm{3}$, and $27 \mathrm{kpc}$ for WR $49=\mathrm{LSS} 2979$, preferring to believe that these two stars either have nonstandard optical colors or dubious photometry. (At these distances, these two stars were outstandingly brighter than all other W-R stars.) The issue of whether there is any distinction between single and binary W-R stars is again worth investigating, in terms of its potential effects on their absolute magnitudes. The magnitudes are summarized in Table 6 on the basis solely of the larger single and binary samples definable from Table 2 using HHASD's spectral types to determine binarity. The single W-R stars seem to be systematically more luminous than the binaries, perhaps suggesting that secondaries interfere with, or even truncate, the W-R stellar winds. However, this effect is not statistically significant: the $\pm 2 \sigma$ bands around the mean colors of single and binary $W-R$ stars are overlapping. Therefore, I have recombined the single and binary $\mathrm{W}-\mathrm{R}$ stars and have broken them down again into WN and WC subtypes ( Table 7). The absolute magnitudes for the set of all WNs are not statistically distinct from those of nondusty WCs, so I further combined the two types into the set of all W-R stars dominated by free-free emission.

To calculate the distances to which an $I R A S$ PSC survey for W-R stars is complete, consider the following. Cohen's (1994) latest version of the SKY model for the point-source sky replicates the pattern of IRAS source counts across the entire sky quite well. By comparing the apparent magnitudes at which the observed IRAS (cumulative) counts depart from SKY's predicted counts, one gains an estimate of the true limits for the onset of IRAS incompleteness as a function of Galactic direction. There are essentially two distinct regimes. In the inner Galaxy, $|l| \leq 30^{\circ},|b| \leq 2^{\circ}$, source surface densities on the sky are so high that $I R A S$ is complete only to $[12] \approx+3.4$ and $[25] \approx+1.8$. Once the latitude exceeds $\sim 2^{\circ}$ from the plane or

TABLE 7

Aasol ute IRAS Magnitudes OF Different TyPes of WOLF-RAYET StaRS

\begin{tabular}{|c|c|c|c|c|}
\hline WR type & $\mathrm{M}_{12}$ & $\mathrm{M}_{25}$ & $\mathrm{M}_{60}$ & $\mathrm{M}_{100}$ \\
\hline WN2 & $-6.13(1)$ & $-8.43(1)$ & $-11.25(1)$ & (0) \\
\hline WN3 & $-5.88 \pm 1.08(5)$ & $-7.28 \pm 1.24(4)$ & $-11.63 \pm 0.31(2)$ & (0) \\
\hline WN4 & $-6.31 \pm 0.25(10)$ & $-8.28 \pm 0.36(6)$ & $-12.85 \pm 2.06(2)$ & $-16.85(1)$ \\
\hline WN4.5 & $-6.73 \pm 0.45(6)$ & $-7.89 \pm 0.75(5)$ & $-10.54 \pm 1.01(3)$ & $-14.67(1)$ \\
\hline WN5 & $-6.21 \pm 0.32(5)$ & $-7.36 \pm 0.33(4)$ & $-9.13 \pm 0.11(2)$ & (0) \\
\hline WN6 & $-7.55 \pm 0.21(17)$ & $-9.00 \pm 0.34(13)$ & $-11.61 \pm 0.63(6)$ & $-17.53(1)$ \\
\hline WN7 & $-7.40 \pm 0.33(11)$ & $-8.93 \pm 0.54(8)$ & $-12.36 \pm 1.09(3)$ & (0) \\
\hline WN8 & $-7.43 \pm 0.26(10)$ & $-9.21 \pm 0.52(10)$ & $-12.21 \pm 1.15(4)$ & $-14.28 \pm 2.42(2)$ \\
\hline WN9 & $-6.96(1)$ & $7.50(1)$ & (0) & (0) \\
\hline All WNs & $-6.99 \pm 0.15(66)$ & $-8.58 \pm 0.21(52)$ & $-11.33 \pm 0.42(24)$ & $-15.52 \pm 0.89(5)$ \\
\hline WC4 & $-6.44 \pm 0.18(2)$ & $-8.40 \pm 0.06(2)$ & $-12.09(1)$ & (0) \\
\hline WC5 & $-6.63 \pm 0.56(10)$ & $-7.46 \pm 0.34(9)$ & $-10.07 \pm 0.80(4)$ & $-15.89(1)$ \\
\hline WC6 & $-6.66 \pm 0.42(9)$ & $-7.84 \pm 0.58(8)$ & $-13.07 \pm 1.01(3)$ & (0) \\
\hline WC7 & $-6.47 \pm 0.23(11)$ & $-7.65 \pm 0.32(9)$ & $-11.32 \pm 1.45(3)$ & $-16.37(1)$ \\
\hline WC8 & $-7.72 \pm 0.37(6)$ & $-8.62 \pm 0.42(6)$ & $-12.27 \pm 2.76(3)$ & $-9.42(1)$ \\
\hline WC9 & $-10.03 \pm 0.51(19)$ & $-10.97 \pm 0.55(17)$ & $-13.96 \pm 0.76(11)$ & $-16.50 \pm 1.62(3)$ \\
\hline All WCs & $-7.85 \pm 0.30(57)$ & $-8.89 \pm 0.31(51)$ & $-12.64 \pm 0.56(25)$ & $-15.20 \pm 1.36(6)$ \\
\hline All DWCLs & $-9.67 \pm 0.52(21)$ & $-10.51 \pm 0.50(21)$ & $-13.95 \pm 0.69(11)$ & $-16.47 \pm 1.15(4)$ \\
\hline All non-dusty WCs & $-6.78 \pm 0.22(36)$ & $-7.77 \pm 0.21(30)$ & $-10.61 \pm 1.06(14)$ & $-12.66 \pm 3.23(2)$ \\
\hline All WRs & $-7.39 \pm 0.16(123)$ & $-8.74 \pm 0.18(103)$ & $-12.00 \pm 0.36(49)$ & $-15.35 \pm 0.81(11)$ \\
\hline All free-free WRs & $-6.91 \pm 0.12(102)$ & $-8.28 \pm 0.16(82)$ & $-11.07 \pm 0.47(38)$ & $-14.70 \pm 1.13(7)$ \\
\hline
\end{tabular}


the longitude is well within the $30^{\circ}-220^{\circ}$ range, then source confusion is far less of a problem, and incompleteness is typically not encountered until $[12] \approx+5.3$ and $[25] \approx+3.7$. To calculate the incompleteness limits for this $I R A S$-based search, one need only establish the maximum volume in which [12] [25] and [25] - [60] are available for an $I R A S$ source within the PSC. At $60 \mu \mathrm{m}$ the density of extended sources (typically $H$ il regions) dictates the degree of confusion in the inner $\mathrm{Gal}-$ axy. However, the great sensitivity of $I R A S$ at this long wavelength, coupled with the intrinsically high W-R luminosities, suggests that the requirement that [25] - [60] be available does not reduce the survey depth determined from the requirement that [12] $-[25]$ exists.

From the absolute magnitudes in Table 7, one therefore concludes that, in the high source density regime, [12] - [25] is complete to $1.0 \mathrm{kpc}$ for free-free-emitting $\mathrm{W}-\mathrm{R}$ stars and to $2.9 \mathrm{kpc}$ for DWCLs. Outside $|\ell|<30^{\circ}$, however, the search is complete to 2.9 and $7.0 \mathrm{kpc}$, for free-free-emitting and dusty W-R stars, respectively. These distances are 1.28 and $3.08 \mathrm{kpc}$ for innermost and outer Galactic regimes if one simply treats all W-R stars as a single class. All of these volumes greatly exceed those associated with optical searches for W-R stars, as anticipated.

\section{CONCLUSIONS}

There is a weak tendency for single W-R stars to be more luminous at $I R A S$ wavelengths than binaries, possibly indica- tive of a truncation of the W-R star's stellar wind by its companion. However, the IRAS attributes (absolute magnitudes and colors) of single and binary nondusty W-R stars are not statistically distinct. I conclude that it is possible to characterize the IRAS colors of both DWCLs and free-free-dominated W-R stars sufficiently sharply that IRAS color-based searches for new W-R candidates of both types are possible. Whether I have been successful can be determined only through followup observations. Either way, this infrared search is complete in a volume much larger than that accessible to optical techniques. Note that Table 5 does suggest potential DWCLs that are not toward the Galactic center. Should any of these stars in the outer Galaxy be shown truly to be a WC 9 star, then the tantalizing idea of Smith \& Maeder (1991), that metallicity governs the Galactic distribution of the WCLs, will have been disproved

This effort was partially supported by NASA under grant NAG 5-2015 with the University of California, Berkeley. I also thank NASA-Ames for partial support under cooperative agreement NCC 2-142 with UC Berkeley. I acknowledge the valuable assistance at IPAC of Debbie Levine, Ann Wehrle, and Sue Terebey in using the SYBASE query language, in obtaining and assembling the many ADDSCANs and FRESCO images, and in using SK YVIEW to process the latter. I am also grateful to Peter Conti for his thorough review of the paper and for making several valuable suggestions.

\section{REFERENCES}

Abbott, D. C. 1982 ApJ. 259.282

Allen, D. A.. Hyland, A. R., Longmore, A. J., Caswell, J. L., Goss, W. M., \& Haynes, R. F. 1977, ApJ, 217, 108

Atlas of Low Resolution IRAS Spectra. 1986, IRAS Science Team, prepared by F. M. Olnon \& E. Raimond, A\&AS, 65, 607 (LRS)

Barlow, M. J., Roche, P. F., \& Aitken, D. K. 1988, MNRAS, 232, 821

Caillault, J.-P., Chanan, G. A., Helfand, D. J., Palterson, J., Nourek, J. A.,

Takalo, L. O., Bothun, A. D., \& Becker, R. H. 1985, Nature, 313, 376

Cohen, M. 1994, AJ, 107, 582

Cohen, M., \& Barlow, M. J. 1975, Astrophys. Lett., 16, 165

Cohen, M., Barlow, M. J., \& Kuhi, L. V. 1975, A\&A, 40, 291

Cohen, M., \& Kuhi. L. V. 1976, PASP, 88, 535

1977a, MNRAS, 180, 37

1977 b, PASP, 89,829

Cohen, M., Tielens, A. G. G. M., \& Bregman, J. D. 1989, ApJ, 344, L13

Cohen, M., van der Hucht, K. A., Williams, P. M., \& Thé, P. S. 1991, ApJ, 378,302

Conti, P. S., \& Vacca, W. D. 1990, AJ, 100,431

Danks, A. C., Dennefeld, M., Wamsteker, W., \& Shaver. P. A. 1983, A\&A. 118,301

Eenens, P. R. J., Williams, P. M., \& Wade, R. 1991, MNRAS, 252,300

Gehrz, R. D., \& Hackwell, J. A. 1974, ApJ, 194, 619

IR IS Point Source Catalog Version 2, Vols. 2-6. 1988, Joint IRAS Science Working Group (NASA RP-1 190; Washington, DC: GPO) (PSC)

Kudritzki, R. P., Pauldrach, A., \& Puls, J. 1987, A\&A, 173, 293

Mathis, J. S., Cassinelli, J. P., van der Hucht. K. A., Prusti, T., Wesselius, P. R., \& Williams, P. M. 1992, ApJ, 384, 197

Morris, P. W. Brownsberger, K. R., Conti, P. S., Massey, P. \& Vacca, W. D. 1993, ApJ, 412, 374

Moshir, M., et al. 1992, Explanatory Supplement to the IRAS Faint Source Survey (Pasadena: JPL)
Price, S. D., \& Walker. R. G. 1976, The AFGL Four Color Infrared Sky Survey (AFGL TR-76-0208)

Shara, M. M., Potter, M., Moffat, A. F. J., \& Smith, L. F. 1991, in IAU Symp. 143, WR Stars and Interrelations with Other Massive Stars in Galaxies, ed. K. van der Hucht \& B. Hidayat (Dordrecht: Kluwer), 591 (SPMS)

Smith, L. F. 1968, MNRAS, 141, 317

Smith, L. F., \& Maeder, A. 1991, A\&A, 241, 77

van der Hucht, K. A. 1991, private communication

van der Hucht, K. A., Hidayat, B., Admiranto, A. G., Supelli, K. R., \& Doom, C. 1988, A\&A, 199, 217 (HHASD)

van der Hucht, K. A., Jurriens, T. A., Olnon, F. M., Thé, P. S., Wesselius, P. R., \& Williams, P. M. 1985a, A\&A, 145, L13

- 1985b, in Birth and Evolution of Massive Stars and Stellar Groups, ed. W. Boland \& H. van Woerden (Dordrecht: Reidel), 167

van der Hucht, K. A., \& Olnon, F. M. 1985, A\& A, 149, L17

Volk, K., \& Cohen, M. 1989, AJ, 98, 931

Volk, K., Kwok, S., Stencel, R. S., \& Brugel, E. W. 1991, ApJS, 77, 607

Volk, K., Stencel, R. S., Brugel, E. W., \& Kwok, S. 1992, private communication

Wainscoat, R. J., Cohen, M., Volk, K., Walker, H. J., \& Schwartz, D. E. 1992, ApJS, 83, 111

Walker, H. J., Cohen, M., Volk, K., Wainscoat, R. J., \& Schwartz, D. E. 1989, AJ, 98,2163

Williams, P. M., Cohen, M., van der Hucht, K., Bouchet, P., \& Vacca, W. D. 1995, MNRAS, in press

Williams, P. M., \& van der Hucht, K. 1992, in ASP Conf. Proc. 22, Nonisotropic and Variable Outflows from Stars, ed. L. Drissen, C. Leitherer, \& A. Nota (San Francisco: ASP), 269

Williams, P. M., van der Hucht, K. A., \& Thé, P. S. 1987, A\&A, 182, 91 (WHT) 